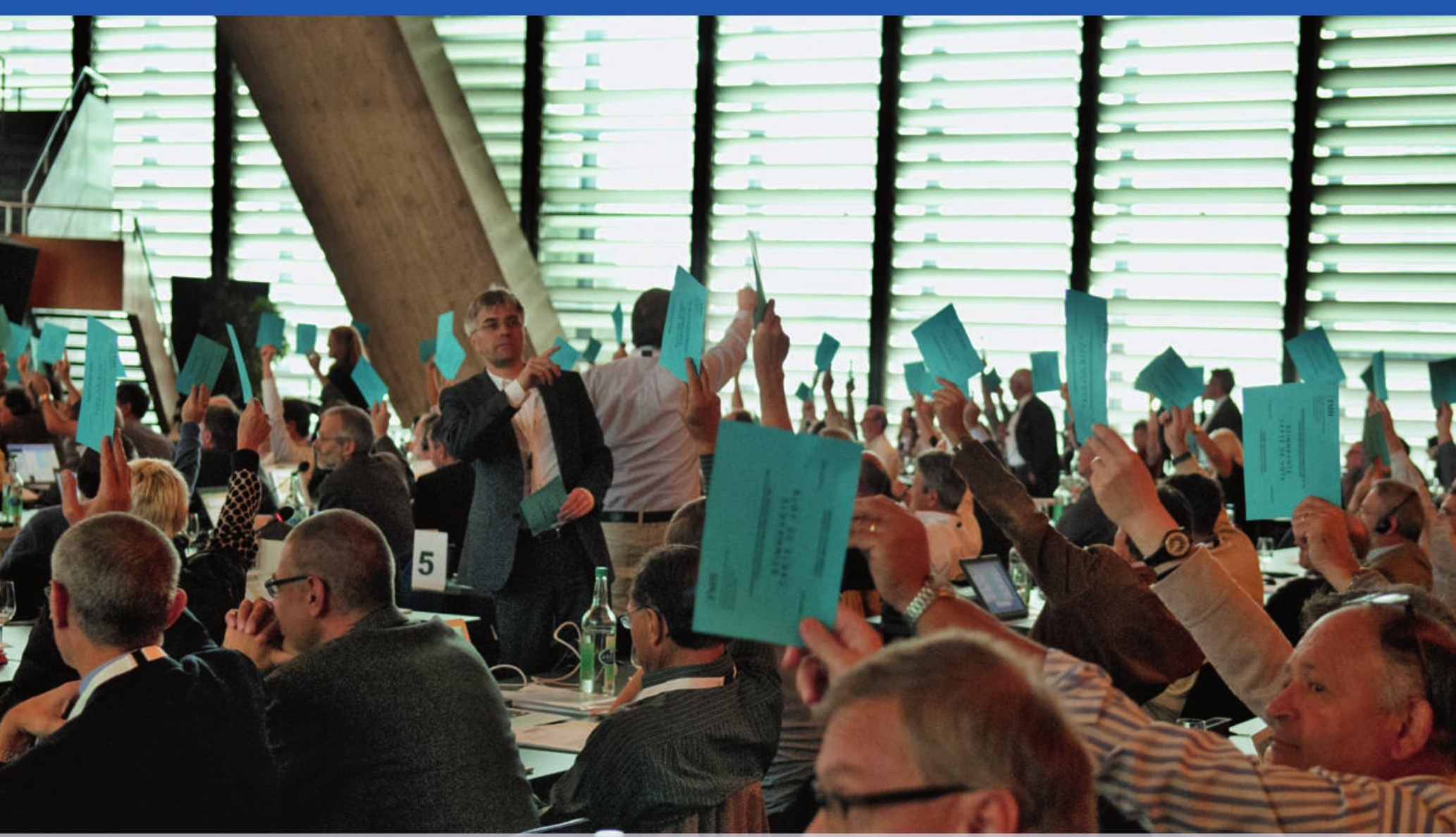

Die Ärztekammer ist das Parlament der FMH und bestimmt die standespolitische Richtung.

Kongresshaus Biel, 3. Oktober 2013

\title{
Protokoll der zweiten Ärztekammer 2013
}

Monika Henzen,

Leiterin Zentrales Sekretariat

\section{Begrüssung, Mitteilungen, Bestellung des Büros}

Jürg Schlup/Präsident begrüsst die Delegierten zur zweiten Ärztekammer 2013 und stellt ihre Beschlussfähigkeit fest. Als Gäste begrüsst er Prof. Frank Ulrich Montgomery, Prof. Peter Tschudi, Bruno Kesseli, Chefredaktor der SÄZ, Hans-Ruedi Koelz, Vizepräsident des SIWF, Rafael Stolz, Vizepräsident SIWF, Rita Zahnd, Geschäftsführerin SGOT-SSOT, Michael Barnikol, Ursina Pally, Gabriela Lang, Anne-Sylvie Thiébaud, alle Rechtsdienst FMH, sowie Michelle Gerber, Ressort DDQ. Entschuldigt ist Beat Bär/FHM-Services.

Anne-Geneviève Bütikofer/Generalsekretärin gibt neben den üblichen organisatorischen Informationen auch Anleitungen zum verteilten Couvert, in welchem sich ein Gerät für die elektronische Abstimmung befindet. Testweise wird heute über die Traktanden 4 und 5 elektronisch abgestimmt. Es wird vorgeschlagen, an der nächsten ÄK-Sitzung und in Zukunft alle Abstimmungen an der ÄK auf diese Weise durchzuführen.

Anschliessend bestellt der Präsident das Büro der ÄK. Dieses besteht aus dem Präsidenten, den Vizepräsi- denten, der Generalsekretärin und den nachfolgenden Stimmenzählenden: Christoph Ramstein/VEDAG, Baseli Werth/GR, Franziska Zogg/ZG, Charles FavrodCoune/VD, Andreas Haefeli/AG, Karl Lövblad/VLSS, Aldo Kramis/LU, Daniel Zwahlen/FG Radioonkologie, Michael Sacristan/VSAO und Daniel Schröpfer/VSAO.

Beschluss: Die Stimmenzählenden werden ohne Gegenstimme gewählt.

\section{Traktandenliste}

Michel Alexandre Matter/GE bemängelt, dass der letzte Versand der Einladung am 30. September 2013 erfolgt ist und die Zeit zu kurz war, um sich insbesondere für Traktandum 8 genügend vorzubereiten. Eine Rücksprache mit der Basis wäre für eine Entscheidfindung wichtig gewesen.

Jürg Schlup/Präsident hat Verständnis für diesen Einwand, erinnert aber daran, dass die Traktandenliste inklusive Traktandum «Hausarztinitiative und Gegenvorschlag» bereits im August 2013, also sechs Wochen vor der ÄK, in der SÄZ publiziert wurde. Der ZV FMH ist der Meinung, dass die statutarische Voraussetzung für die Behandlung dieses Traktandums erfüllt ist. Die Hausarztinitiative wurde erst am 
27. September 2013 zurückgezogen, und das Parlament hat gleichentags den Gegenvorschlag verabschiedet. Er macht beliebt, das Traktandum beizubehalten.

Daniel Schröpfer/VSAO hätte eine vertieftere Diskussion gewünscht und schlägt vor, für die Mitglieder Stimmfreigabe zu beschliessen. Jürg Schlup/Präsident hält fest, dass es hier um die Genehmigung der Traktandenliste geht. Eine allfällige Abstimmung über Stimmfreigabe kann unter Traktandum 8 eingefordert werden. Ergänzend hält er fest, dass die mündliche Information Traktandum 11 vom Vorsitzenden der DV zurückgezogen wurde.

Beschluss: Die Traktandenliste wird mit $110 \mathrm{Ja}$ zu 6 Nein genehmigt.

Der Präsident beantragt, die ÄK - wie in den Statuten vorgesehen - spätestens um 17.00 Uhr zu beenden. Gemäss Art. 11 Abs. 3 der Geschäftsordnung legt er fest, dass ab 17.00 Uhr keine Beschlüsse mehr gefasst und auch keine Wahlen mehr vollzogen werden.

Beschluss: Der Antrag wird einstimmig gutgeheissen.

\section{Eingangsreferat Präsident FMH}

In seinem Eingangsreferat geht der Präsident auf die Wiedereinführung des Zulassungsstopps für Ärztinnen und Ärzte sowie die personalisierte Medizin und deren Auswirkungen ein.

Beim Zulassungsstopp haben die FMH, der VSAO und mit ihnen die gesamte Ärzteschaft einen grossen Erfolg erkämpft. Die FMH konnte in den letzten 10 Jahren weder die Einführung des Stopps noch dessen zweimalige Verlängerung verhindern. Sie

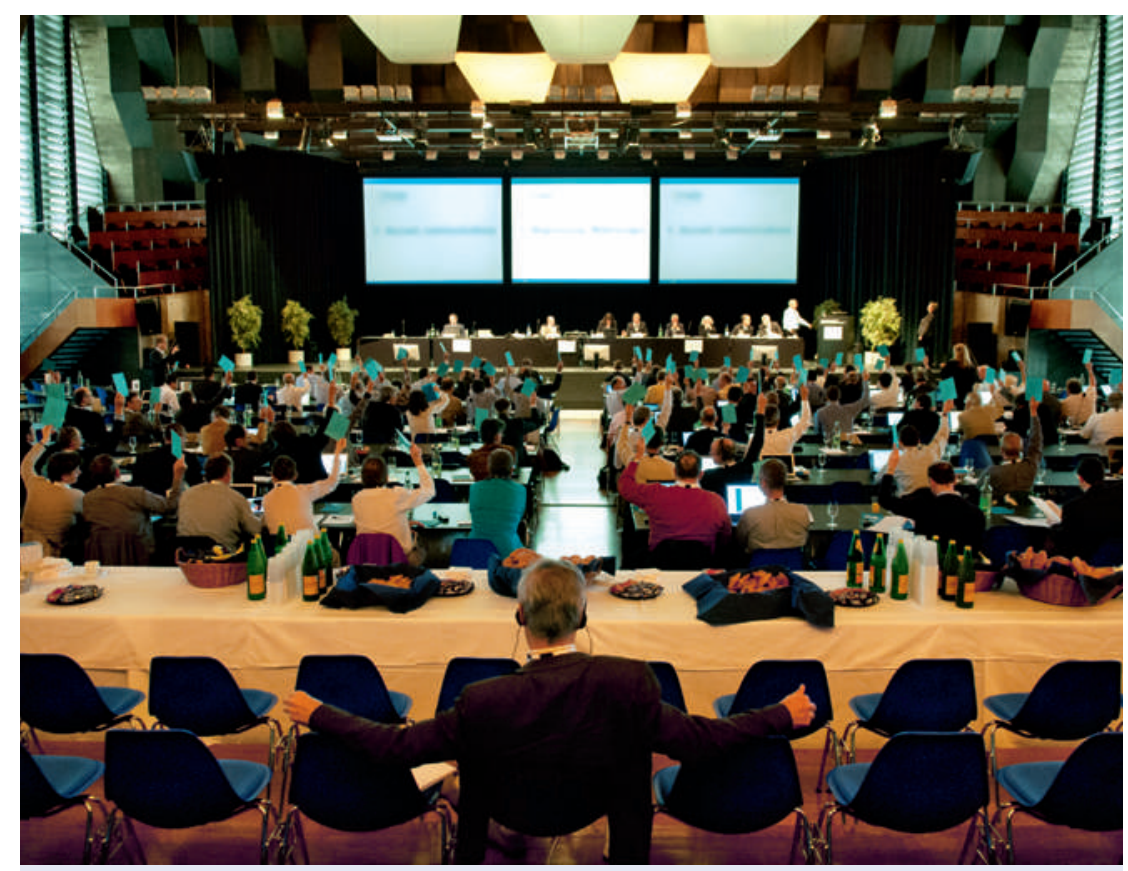

Mit 200 stimmberechtigten Delegierten bildet die Ärztekammer - neben der Urabstimmung das oberste Organ der FMH. konnte jetzt jedoch die Wiedereinführung des Zulassungsstopps derart entschärfen, dass dieser heute für 90\% der berufstätigen Ärztinnen und Ärzte nicht anwendbar ist und bei in der Schweiz weitergebildeten Ärztinnen und Ärzten nicht gilt. Der Präsident dankt im Namen des Vorstandes allen Mitgliedern der FMH, allen involvierten Parlamentariern sowie den Professoren für Europarecht für ihre Unterstützung.

Aktuelle tiefgreifende Veränderungen in der Gesellschaft und in der Medizin sind auch für die Ärzteschaft eine wichtige Herausforderung, die nicht zu unterschätzen ist. Am Beispiel der internetbasierten Gentests - den sogenannten Consumer Genomics wird die Bedeutung für die Ärzteschaft aufgezeigt. Die vermehrte Inanspruchnahme solcher spontaner Gentests durch die Bevölkerung und die Erweiterung genetischer Untersuchungen weg von der streng medizinischen Indikation hin zum Konsumgut hat die Expertenkommission für genetische Untersuchungen beim Menschen (GUMEK) dazu bewegt, ihre Befürchtungen bezüglich Versorgungssicherheit in der medizinisch-genetischen Diagnostik in einem Schreiben an Bundesrat Alain Berset anzusprechen. Die GUMEK hat mit der Schweizerischen Konferenz der Kantonalen Erziehungsdirektoren (EDK) Kontakt aufgenommen und sie auf verschiedene Probleme in diesem Zusammenhang aufmerksam gemacht.

Was bedeutet diese Entwicklung für die Ärzteschaft? An einer Ärztefortbildung im Mai dieses Jahres vertraten die anwesenden zumeist praktizierenden Ärztinnen und Ärzte allgemein die Auffassung, auf absehbare Zeit wären vermehrte Tests auf genetische Risiko-Faktoren kein Thema und daher auch keine zusätzliche Belastung. Dies, weil ein genetischer Test nur bei wenigen konkreten Indikationen verordnet würde und auch nur in diesen Fällen therapieentscheidend wirksam werden könne. Zwei Wochen danach schrieb eine grosse KonsumentenZeitschrift («Beobachter») einen Artikel zum Thema Gentests mit folgendem Titel: «Gentests: Die Versicherungen warten schon auf Ihre Daten.»

Der Präsident ist der Meinung, dass, sobald der Preis für eine DNS-Sequenzierung unter 500 US-Dollar sinken wird, viele Ärzte hilfesuchende Menschen nach freiwillig, spontan und privat durchgeführten Gentests - beraten und behandeln müssen. Wenn die ärztliche Hilfe nicht erfolgt, verweisen die Ärzte diese Patienten damit möglicherweise auf eine nicht-ärztliche Beratung.

Wäre eine nicht-ärztliche Beratung vor oder nach der Durchführung eines privaten Gentests im Interesse der betroffenen Menschen oder unserer Mitglieder?

Diese Fragestellungen sind ein Beispiel dafür, welche Entwicklungen im Gang sind und wie unterschiedlich die Bereitschaft unserer Mitglieder ist, solche Entwicklungen wahrzunehmen. Jürg Schlup/Präsident appelliert an die Verantwortung jedes Einzelnen und die Aufgabe der ÄK-Delegierten, unsere 
Mitglieder auf diese Entwicklung aufmerksam zu machen.

\section{2. Änderungen in den Statuten und in der Geschäftsordnung}

\subsection{Statutenergänzung und Revision Anhang 1 der Geschäftsordnung: \\ «Arztpraxen von ordentlichen Mitgliedern» als juristische Personen}

Hanspeter Kuhn/FMH erläutert den Antrag des ZV. Die Arbeitgeber müssen sich für die Erfüllung ihrer AHV/IV/EO/ALV-Beitragspflicht einer Ausgleichskasse anschliessen. Für Mitglieder der FMH, SSO, GST und SCG ist die Ausgleichskasse medisuisse zuständig. Den Verbandsausgleichskassen werden alle Arbeitgeber und Selbständigerwerbenden angeschlossen, die einem Gründerverband angehören. Diese Bedingung ist erfüllt, wenn der selbständigerwerbende Arzt FMH-Mitglied ist. Ist die Arztpraxis hingegen in der Rechtsform der juristischen Person organisiert, haben einige kantonale Ausgleichskassen begonnen, die Praxis aufzufordern, ihr Personal bei der kantonalen Ausgleichskasse zu versichern. Es ist anzustreben, dass die FMH-Mitglieder ihre Arztpraxis unabhängig von der gewählten Rechtsform weiterhin bei medisuisse versichern können.

Der Vorschlag für die Ergänzung von Statuten und Geschäftsordnung sieht deshalb vor, dass ordentliche FMH-Mitglieder ihre als juristische Person organisierte Arztpraxis bei der FMH anmelden können, wenn Eigentum, strategische und operative Führung des Unternehmens in der Hand von ordentlichen FMH-Mitgliedern liegen, also bei der Praxis als AG Aktien, Verwaltungsrat und Geschäftsführung ausschliesslich in ihren Händen sind. Das Bundesamt für Sozialversicherungen hat die vorliegende Lösung akzeptiert.

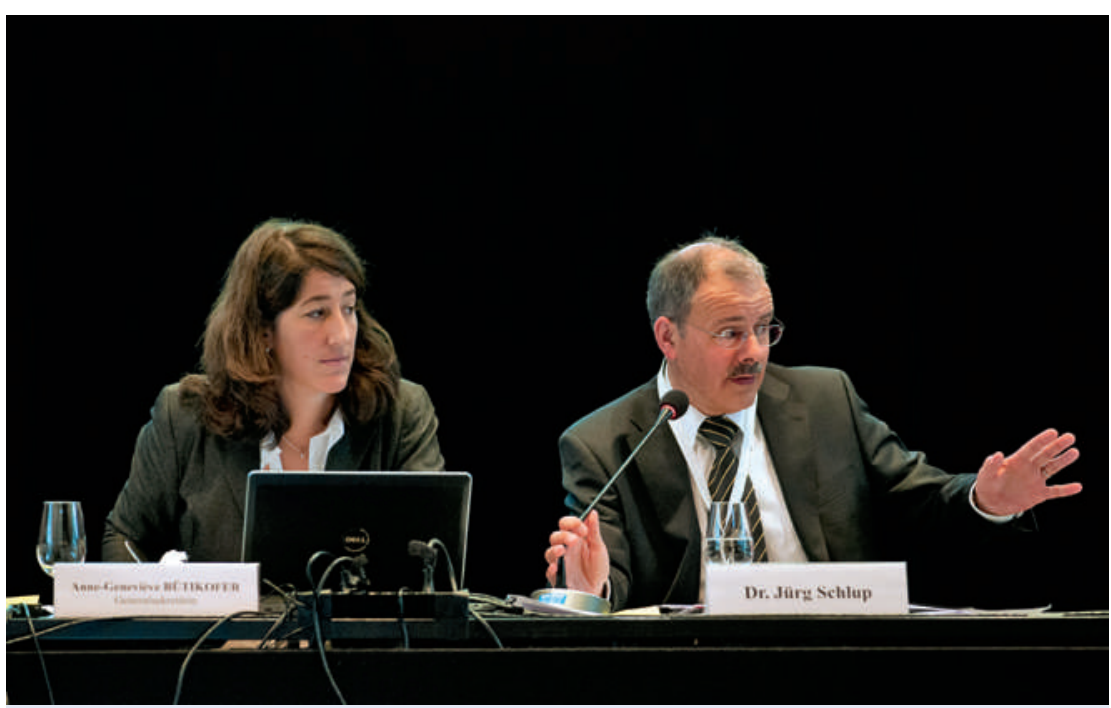

Dr. med. Jürg Schlup, FMH-Präsident, und Frau Anne-Geneviève Bütikofer, Generalsekretärin, moderieren gemeinsam die Grossveranstaltung.
Weil die FMH Gründerorganisation der Verbandsausgleichskasse ist und somit für das AHVRecht ausschliesslich die FMH-Mitgliedschaft massgeblich ist, sieht Art. 5bis der Geschäftsordnung vor, dass das Generalsekretariat die Unterlagen prüft, den Anschluss der Arztpraxen von ordentlichen Mitgliedern als juristische Personen bestätigt sowie Rechnungsstellung und Inkasso besorgt.

Andreas Haefeli/AG hat zu diesem Geschäft einen Gegenantrag eingereicht, den er den Anwesenden erläutert. Er unterstützt den Vorschlag des ZV in der Zielrichtung, wünscht jedoch folgende Änderung: Ordentliche Mitglieder können ihre als juristische Person organisierte Arztpraxis der FMH anschliessen, wenn Eigentum und oberste operative Führung ausschliesslich, die strategische Führung mehrheitlich in ihren Händen liegt.

Wenn diesem Vorschlag zugestimmt wird, müsste folgerichtig auch Art. 5bis der Geschäftsordnung angepasst werden.

Nach ihm bestimmt die operative Führung selber, was die angestellten Ärzte oder die Angestellten zu tun oder nicht zu tun haben. Deshalb ist er für die Formulierung, dass die operative Führung «ausschliesslich» dafür verantwortlich ist. Die strategische Führung hat eine andere Aufgabe. Die entscheidet zum Beispiel über Bau und Weiterentwicklung einer Praxis. Das sind Entscheidungen, die nicht direkt ins Arzt-Patienten-Verhältnis eingreifen.

Aus Sicht von Hanspeter Kuhn/FMH stellt der Vorschlag von Andreas Haefeli kein Problem dar. Für ihn ist es eine Frage der politischen Würdigung, welche Lösung bevorzugt wird.

Jürg Rufener/SGIM ist der Meinung, dass das zweite Kriterium aufgeweicht werden müsste, und stellt den Antrag, dass Eigentum «mehrheitlich», die operative und strategische Führung «ausschliesslich» in ärztlichen Händen liegen soll. Weiter findet er, dass das Kriterium entweder mit «grossmehrheitlich» oder mit einem Prozentsatz zu umschreiben ist.

Ricardo Andreas Torriani/ZH informiert, dass sich ihr Vorstand für mehrheitliche Besitzverhältnisse entschieden habe, und zwar sowohl aus den bereits gehörten Gründen wie aber auch aufgrund der Tatsache, dass bei Übergangslösungen häufig die bestehenden alten Praxisinhaber teilweise Kapital im Unternehmen belassen und nicht mehr praktizieren.

Hanspeter Kuhn/FMH ist der Meinung, dass sich die ÄK auch zu den Besitzverhältnissen für die sinnvollere Lösung entscheiden kann. Er geht nicht davon aus, dass für die Genehmigung des Bundesamtes für Sozialversicherungen eine Mehrheitsbeteiligung als Kriterium ein Problem wäre. Er hält fest, dass «ausschliesslich» 100\% und «mehrheitlich» 51\% bedeuten.

Angelo Cannova/ZH plädiert für ausschliesslich und nicht für mehrheitlich. Er ist dafür, dass nur Ärzte, also Organisationen, die nur von Ärzten geführt werden, angeschlossen sind. 
Für Philippe Vuillemin/VD ist vielmehr die strategische Entscheidung als die operative Leitung wesentlich. Deshalb ist der Satz nochmals zu überdenken und neu zu formulieren, weil operativ und strategisch sonst falsch verstanden werden. Die Strategie muss ausschliesslich sein, die operative Führung eventuell mehrheitlich.

Pierre Vallon/FMPP stellt den Ordnungsantrag, die Diskussionen auch aufgrund des reichbefrachteten Programms zu beenden.

Beschluss: Dem Ordnungsantrag wird grossmehrheitlich stattgegeben.

Das Abstimmungsprozedere wird festgelegt. Über die Anträge wird wie folgt abgestimmt:

Antrag Haefeli: «Ordentliche Mitglieder können ihre als juristische Person organisierte Arztpraxis der FMH anschliessen, wenn Eigentum und oberste operative Führung ausschliesslich, die strategische Führung mehrheitlich in ihren Händen liegt.»

Beschluss: Der Antrag wird mit 52 Ja zu 62 Nein und 21 Enthaltungen abgewiesen.

Antrag Rufener: Das Eigentum liegt «mehrheitlich» in ärztlicher Hand und die operative und strategische Führung «ausschliesslich» in ärztlicher Hand.

Beschluss: Der Antrag wird mit $65 \mathrm{Ja}$ zu 41 Nein mit 30 Enthaltungen angenommen.

Gemäss Antrag Rufener modifizierter Antrag des ZV für die Schlussabstimmung: Ordentliche Mitglieder können ihre als juristische Person organisierte Arztpraxis der FMH anschliessen, wenn die Eigentumsrechte mehrheitlich und die strategische und operative Führung ausschliesslich in ihren Händen liegt.

Beschluss: Der Antrag des ZV wird mit $111 \mathrm{Ja}$ zu 5 Nein angenommen.

\subsection{Revision Geschäftsordnung Art. 21:}

\section{Amtsantritt ZV und Präsident}

Ursina Pally/FMH präsentiert den neuen Vorschlag. Dieser enthält die Anforderungen, welche vom ZV und auch von der DV an den Amtsantritt der ZVMitglieder und den Präsidenten gestellt werden. Es soll wie bis anhin möglich sein, dass die Gewählten ihre Wahl sofort, also am Ende der Sitzung, antreten können. Damit die Neugewählten ihre Angelegenheiten regeln können, wird neu eine Frist eingeräumt. Diese dauert nach der Frühjahrssitzung bis zum 1.7. desselben und im Herbst bis zum 1.1. des folgenden Jahres. Da es nicht immer möglich ist, dass das bisherige Mitglied so lang im Amt bleiben kann, bis das neue Mitglied übernimmt, gibt es eine Ausnahmeregelung. Diese kommt nur zum Tragen, wenn das neugewählte Mitglied oder der neugewählte Präsident einen Antrag an die ÄK stellt. Die Regelung erlaubt eine Übernahme bis ein halbes Jahr nach der Wahlsitzung, nach Ende der Wahlsitzung. Hier gibt es aber eine Ausnahme: Der neugewählte Präsident muss die nächste ÄK in jedem Fall leiten.
Der Amtsantritt sollte grundsätzlich so früh als möglich sein.

Philippe Vuillemin/VD dankt dem ZV für die Einreichung dieses Antrages. Die Frage ist für ihn nach wie vor, ob ein an der Mai-ÄK neugewähltes Mitglied sein Amt bereits am 1.7. antreten kann. Er würde die Formulierung in Art. 2 Abs. 2 «auf Wunsch der neuen Mitglieder des ZV oder des neuen Präsidenten» begrüssen. Der Handlungsspielraum sollte nicht mit dem Wort ausnahmsweise eingeschränkt werden.

Konrad Schiess/SG stellt den Antrag, Abs. $2 \mathrm{zu}$ streichen. Ein Präsident soll nach seiner Wahl seine Funktion sofort antreten. Kann er dies nicht tun, besteht immer noch die Möglichkeit, dem Vizepräsidenten weitere Aufgaben zu delegieren, damit der Präsident in einer Übergangsphase entlastet werden kann.

Vincenzo Liguori/TI: Es geht auch um Honorare. Entschädigungen oder Rückforderungen bei einer Doppelbelegung müssen klar geregelt sein.

Ursina Pally/FMH stellt klar, dass in der heutigen Regelung ein Übergabezeitpunkt vorgesehen ist, welcher eine Doppelbelegung und ein Doppelhonorar ausschliesst.

Das Abstimmungsprozedere wird festgelegt. Es wird über zwei Anträge abgestimmt:

Antrag Schiess: Streichung Absatz 2 des Änderungsvorschlages. Der Absatz 2 beginnt «In Ausnahmefällen und auf Antrag des neuen ZV-Mitglieds oder des neuen Präsidenten ...».

Beschluss: Der Antrag wird mit 85 Nein zu 39 Ja mit 4 Enthaltungen abgelehnt.

Antrag ZV: Art. 21 Wahl neu:

${ }^{1}$ Der Amtsantritt eines ZV-Mitglieds oder eines Präsidenten erfolgt bei Wahl an der Frühjahrssitzung spätestens am 1.7. desselben Jahres, bei Wahl an der Herbstsitzung spätestens am 1.1. des darauffolgenden Jahres. Der Zeitpunkt der Amtsübergabe kann vom neuen $Z V$ Mitglied oder vom neuen Präsidenten zwischen dem Ende der Sitzung, an welcher gewählt wurde, und dem 1.7. bzw. dem 1.1. frei bestimmt werden. Das bisherige ZV-Mitglied oder der bisherige Präsident bleibt im Amt, bis dieses vom Nachfolger angetreten wird.

2 In Ausnahmefällen und auf Antrag des neuen ZV-Mitglieds oder des neuen Präsidenten kann die Ärztekammer für den Amtsantritt einen späteren als den in Abs. 1 vorgesehenen Zeitpunkt bestimmen. Dieser Zeitpunkt darf auf längstens 6 Monate nach der Wahl festgesetzt werden, wobei der neue Präsident die auf die Wahl folgende Ärztekammer in jedem Fall zu leiten hat.

${ }^{3}$ Die ZV-Mitglieder und der Präsident teilen dem ZV und GPK innert 14 Tagen nach ihrem Amtsantritt ihre Ämter und Funktionen gemäss Art. 22a der Statuten mit. Sie melden Änderungen ihrer Ämter und Funktionen innert 14 Tagen.

Beschluss: Der Antrag wird mit 127 Ja mit 3 Enthaltungen gutgeheissen. 
2.3 Revision Geschäftsordnung Anhang 1: Mitgliederkategorien und Beitragskategorien

\subsubsection{Neuformulierung Mitglieder-}

kategorien 1-4

Die Mitgliederstruktur der FMH hat sich seit der Strukturreform 1998 verändert und wird sich auch in Zukunft ändern. Die aktuellen Mitgliederkategorien entsprechen nicht mehr in allen Punkten den heutigen Gegebenheiten, insbesondere berücksichtigt die Umschreibung der Kategorie 1 noch nicht, dass viele Ärzte heute ihre Praxis als juristische Person betreiben.

Hanspeter Kuhn/FMH erläutert die wesentlichen Änderungen und neuen Vorschläge.

Kategorie 1: Änderung

Es wird nicht mehr von «selbständig tätigen Ärztinnen und Ärzten» gesprochen, sondern von praktizierenden Ärztinnen und Ärzten. Es wird folgende Formulierung vorgeschlagen: «Vorwiegend ausserhalb des Spitals rechtlich oder faktisch medizinisch eigenverantwortlich tätige Ärztinnen und Ärzte inkl. vorwiegend ambulant tätige Belegärzte, in der Regel mit eigener Berufsausübungsbewilligung, AHV-rechtlich selbständig oder angestellt.»

Kategorie 2: Änderung

Es wird nicht mehr von unselbständig tätigen Ärztinnen und Ärzten in leitenden Funktionen gesprochen, sondern von leitenden Spitalärztinnen und Spitalärzten. Bei den Belegärzten soll das Kriterium sein, ob sie mehr Zeit im Spital oder in der Praxis verbringen, je nachdem gehören sie zur Kategorie 2 oder 1.

Kategorie 3: Änderung

Umbenennung von unselbständig tätigen Ärztinnen und Ärzten in nicht leitender Funktion auf im Spital tätige Oberärztinnen und -ärzte und Spitalfachärztinnen und -ärzte. Es geht um die Oberarzt-Kategorie und die Spitalfachärztinnen.

Kategorie 4: Änderung

Angesichts der heutigen Weiterbildungsorganisation wird nicht mehr von Ärztinnen und Ärzten in Weiterbildung zu einem eidgenössischen Facharzttitel gesprochen, sondern von Ärztinnen und Ärzten in Weiterbildung zu einem Facharzttitel oder Schwerpunkt.

Philipp Jenny/SGP stellt den Antrag, die Kategorien 1, 2 und 3 zusammenzufassen. Er ist der Meinung, dass alle Ärzte in der Schweiz einen FMH-Titel tragen und nicht mehr in Weiterbildung sind. Die veralteten Kategorien sind zu streichen und in eine gemeinsame Kategorie überzuführen.

Hanspeter Kuhn/FMH gibt hier zwei Fragen zu bedenken. Erstens: Welche Art von Statistik soll bei der FMH geführt werden? Eine Unterscheidung ist sicher sinnvoll. Zweitens: Welche Mitgliederbeiträge sollen sachgerecht erscheinen?

Daniel Schröpfer/VSAO: Falls der Antrag des ZV angenommen wird, stellt der VSAO den Gegenantrag, die Kategorien 1, 2 und 3 nicht zusammenzufassen. Der VSAO findet die Zusammenführung der
Spitalfachärztinnen und Oberärzte als Kategorie 3 nicht sinnvoll, weil man als Oberarzt noch in einer Ausbildung ist, während Spitalfachärzte selbständig arbeiten.

Hans-Jakob Riedtmann/ZH stellt den Antrag, das Geschäft an den ZV zur Überarbeitung zurückzuweisen. Einerseits wegen der unselbständig arbeitenden Ärzte in ambulanter Praxis, andererseits wegen der Frage, die der VSAO soeben aufgeworfen hat. Die Vorlage ist unausgereift.

Jürg Schlup/Präsident entgegnet, dass die Vorlage bereits zweimal in der DV behandelt und auch verabschiedet wurde.

Hans Ulrich Iselin/AG stellt einen Ordnungsantrag. Er will die Vorlage nun verabschieden. Für ihn umfasst Kategorie 1 alles, was bereits diskutiert wurde, und weitere Diskussionen sind zu beenden.

Es wird über den Ordnungsantrag abgestimmt:

Beschluss: Dem Ordnungsantrag wird mehrheitlich zugestimmt.

Das Abstimmungsprozedere wird festgelegt. Über die Anträge wird wie folgt abgestimmt:

Antrag Riedtmann: Rückweisung an den ZV.

Beschluss: Der Antrag wird mit 95 Nein zu 32 Ja mit 6 Enthaltungen zurückgewiesen.

Antrag Jenny: Zusammenlegung Kategorien 1, 2 und 3.

Beschluss: Der Antrag wird mit 133 Nein zu 4 Ja und 1 Enthaltung zurückgewiesen.

Antrag Schröpfer: Trennung der Oberärzte von den Spitalfachärzten aufgrund der Funktionsausübung und des Verdienstes. Die Oberärzte sind nach wie vor noch in Ausbildung, und die Spitalfachärzte arbeiten selbständig. Deshalb wird die Einführung der Kategorien $3 a$ und $3 b$ empfohlen.

Beschluss: Der Antrag wird mit 92 Nein zu 41 Ja und 8 Enthaltungen zurückgewiesen.

Antrag ZV: Neuformulierung Mitgliederkategorien 1 bis 4 .

Beschluss: Der Antrag wird mit 110 Ja zu 26 Nein angenommen.

\subsubsection{Anpassung Beiträge für Mitglieder-}

kategorie 3

Hanspeter Kuhn/FMH erläutert die Vorschläge des ZV.

Der ZV wie die DV haben versucht, die veränderten Arbeitsmodelle in den Anträgen aufzunehmen. Eine zunehmend grosse Anzahl von Ärztinnen und Ärzten sieht ihre berufliche Zukunft in einer unselbständigen angestellten Tätigkeit, in nicht leitender Funktion, in Teilzeittätigkeit. Somit ist die Kategorie 3 nicht mehr als Durchgangskategorie, sondern als permanente Mitgliederkategorie zu betrachten. Der ZV empfiehlt die Anpassung des Mitgliederbeitrags Kategorie 3 von 1/2 bisher auf neu 2/3.

Für Daniel Schröpfer/VSAO dient diese Beitragserhöhung der Verringerung des FMH-Defizits. Der VSAO ist gegen eine solche Erhöhung. Er erinnert an die ÄK-Beschlüsse von 2006 und 2009, wo man bereits auf Kosten der Assistenz- und Oberärzte die Rückerstattung beim Titelerwerb eingestellt hat. 
Weiter ist nicht klar, welche aktuellen Veränderungen eine Erhöhung der Beiträge rechtfertigen. Sollte die Beitragserhöhung in der Ärztekammer beschlossen werden, stellt der VSAO den Antrag, dass die Mehrkosten, für die diese Erhöhung gedacht ist, ausgewiesen werden müssen.

Philipp Jenny/SGP erwähnt, dass die unselbständig tätigen Ärzte, die in der Praxis arbeiten, bereits in die Kategorie 1 transferiert wurden, so dass diese Argumentation, wer mehr oder weniger Mitgliederbeitrag zahlt, nicht mehr gilt. Er wünscht eine Begründung, warum jetzt ein unselbständig Tätiger in der freien Praxis anders als ein unselbständig Tätiger im Spital gesehen wird.

Hanspeter Kuhn/FMH stellt fest, dass das Argument von Daniel Schröpfer zu einem gewissen Ausmass noch zutrifft, das heisst, dass es dem ZV und der DV in den Diskussionen bisher sachgerecht erschien, einen Unterschied zwischen der Oberarztfunktion und der Funktion in der Arztpraxis bestehen zu lassen. Aber das sind politische Entscheidungen, das ist nicht ein rechtlich zwingendes Argument.

Ricardo Torriani/ZH ist der Meinung, dass bei einer konsequenten Umsetzung das steuerbare Einkommen als Grundlage herbeigezogen werden muss. Es ist unverständlich, warum ein unselbständig tätiger Arzt, der gleich viel wie ein Selbständiger von der FMH profitiert, weniger zahlen sollte.

Für Anja Zyska Cherix/VSAO ist das aufgeführte Argument für eine Erhöhung der Kategorie 3 falsch. Die Klinikchefs - in der Romandie zumindest - haben keine Daueranstellung, sondern wechseln nach wenigen Jahren.

Philipp Jenny/SSG ist gleicher Meinung. Die heutige Kategorieneinteilung hat nichts mehr mit dem Mitgliederbeitrag zu tun, wenn man sich nur auf die Finanzen bezieht. Betreffend Haftung des Mitgliederbeitrages stellt er den Antrag, dass statt $2 / 3$ neu $1 / 1$ steht, also dass diese dritte Kategorie den gleichen Beitrag wie alle anderen zahlt.

Daniel Schröpfer/VSAO bezieht sich auf seinen Vorredner und stellt die Frage, ob sich der Kollege bewusst ist, dass die Oberärzte und Oberärztinnen in den Spitälern noch eine Weiterbildung erfahren und damit durchaus nicht dieselben finanziellen Ressourcen wie zum Beispiel leitende Ärzte und Chefärzte haben. Deshalb muss der Unterschied - egal, ob man das von der Verantwortungsseite oder von der finanziellen Seite sieht - bestehen bleiben.

Ricardo Torriani/ZH stellt den Antrag, die Mitgliederbeiträge auf dem steuerbaren Einkommen zu berechnen.

Bernhard Sorg/ZH stellt den Ordnungsantrag auf Beendigung der Diskussionen.

Beschluss: Dem Ordnungsantrag wird mehrheitlich zugestimmt.

Das Abstimmungsprozedere wird festgelegt. Über die Anträge wird wie folgt abgestimmt:
Antrag Schröpfer: Der VSAO beantragt die Beibehaltung des Beitrages von 1/2. Sollte die Ärztekammer eine Steigerung auf $2 / 3$ wünschen, sollen die Mehrkosten ausgewiesen werden.

Beschluss: Der Antrag wird mit 96 Nein zu 42 Ja und 3 Enthaltungen abgelehnt.

Antrag Jenny: Die Mitglieder der Kategorien 3 zahlen den gleichen Betrag wie jene der Kategorien 1 und 2, nämlich den vollen.

Beschluss: Der Antrag wird mit 134 Nein zu 14 Ja abgelehnt.

Antrag Torriani: Die Mitgliederbeiträge sind gemäss dem steuerbaren Einkommen zu berechnen. Somit sind alle Sonderfälle und alle zu 50, 45 und 33\% tätigen Ärztinnen und Ärzte eingeschlossen. Als Grundlage ist das aus ärztlicher Tätigkeit erzielte Einkommen massgebend.

Beschluss: Der Antrag wird mit 117 Nein zu 25 Ja und 5 Enthaltungen abgelehnt.

Antrag ZV: Anpassung des Beitrags in der Kategorie 3 von $1 / 2$ auf $2 / 3$.

Beschluss: Der Antrag wird mit 98 Ja zu 45 Nein und 5 Enthaltungen angenommen.

\subsection{Anpassung Statuten und Geschäftsordnung:} Umbenennung «Ressort» zu «Departement» Anne-Geneviève Bütikofer/GS erläutert die Neubezeichnungen. Die Bezeichnung der Arbeitseinheiten im Generalsekretariat ist nicht eindeutig und führt zu Missverständnissen. Im Rahmen der Umstrukturierung hat sich eine Vereinheitlichung der Bezeichnungen als erforderlich erwiesen. Im Übrigen wurde die Verwendung des Begriffs «Ressort» im Französischen wie im Deutschen zur Bezeichnung der von einem Mitglied des Zentralvorstands abhängigen politischen Einheit von den Delegierten der ÄK mehrfach kritisiert und verlangte nach einer Revision. Eine Zustimmung der neuen Bezeichnung erfordert eine Anpassung in den Statuten sowie in der Geschäftsordnung.

$\begin{array}{ll}\begin{array}{l}\text { Bisher } \\ \text { Ressortverantwortliche/r } \\ \text { (ZV-Mitglied) }\end{array} & \begin{array}{l}\text { Neu } \\ \text { Departementsverantwortliche/r } \\ \text { (ZV-Mitglied) }\end{array} \\ \begin{array}{l}\text { Leiter/in Ressort } \\ \text { oder Leiter/in Abteilung }\end{array} & \text { Leiter/in Abteilung } \\ \text { Leiter/in Dienst } & \text { Leiter/in Dienst }\end{array}$

Pierre Vallon/FMPP will wissen, ob es Departemente gibt mit mehr als einer Leitung.

Jürg Schlup/Präsident erwähnt die Ressorts Tarifbereich und eHealth. Diese beiden haben jeweils zwei Leiter.

Es wird über folgenden Antrag abgestimmt:

Antrag ZV: Gutheissung der neuen Bezeichnung und Anpassung der Änderung in den Statuten und in der Geschäftsordnung.

Beschluss: Der Antrag wird grossmehrheitlich angenommen. 


\section{Gastreferat}

Als Gastreferent spricht Prof. Dr. Frank Ulrich Montgomery, Präsident der Bundesärztekammer Deutschland. Er vertritt 430000 Mitglieder und lobt einleitend die sehr gute Zusammenarbeit mit der FMH in vielen europäischen Gremien. Professor Montgomery vermittelt einen gerafften Einblick in die aktuellen wie auch äusserst komplexen Fragestellungen rund um das Gesundheitswesen, die teilweise auch in der Schweiz immer wieder neu debattiert werden. Der Referent beleuchtet verschiedenste Aspekte, welche die Ärzteschaft, die Patienten, die Politik und die Öffentlichkeit insgesamt beschäftigen.

Das Gesundheitswesen ist in Deutschland ein grosser Beschäftigungssektor, mit 300 Milliarden Euro Gesamtausgaben grösser als die Automobilindustrie. Das Gesundheitswesen in Deutschland zeichnet eine hohe Qualität aus. Gemäss einer Untersuchung haben sie die kürzesten Behandlungswartezeiten, die schnellsten Befunde, die niedrigste Infektionsrate, die geringste Wiederaufnahmerate und die beste Versorgung chronisch Kranker. Sie haben jedoch ein sehr schlechtes Entlassungsmanagement. Dennoch sieht die «deutsche Volksseele» das ganz anders. Trotz dem erwähnten hohen Behandlungsniveau ist die Patientenzufriedenheit gering.

Obwohl Deutschland objektiv wohl ein gutes System hat, braucht es Reformen. Das heutige Umlageverfahren muss zwingend geändert werden. Die

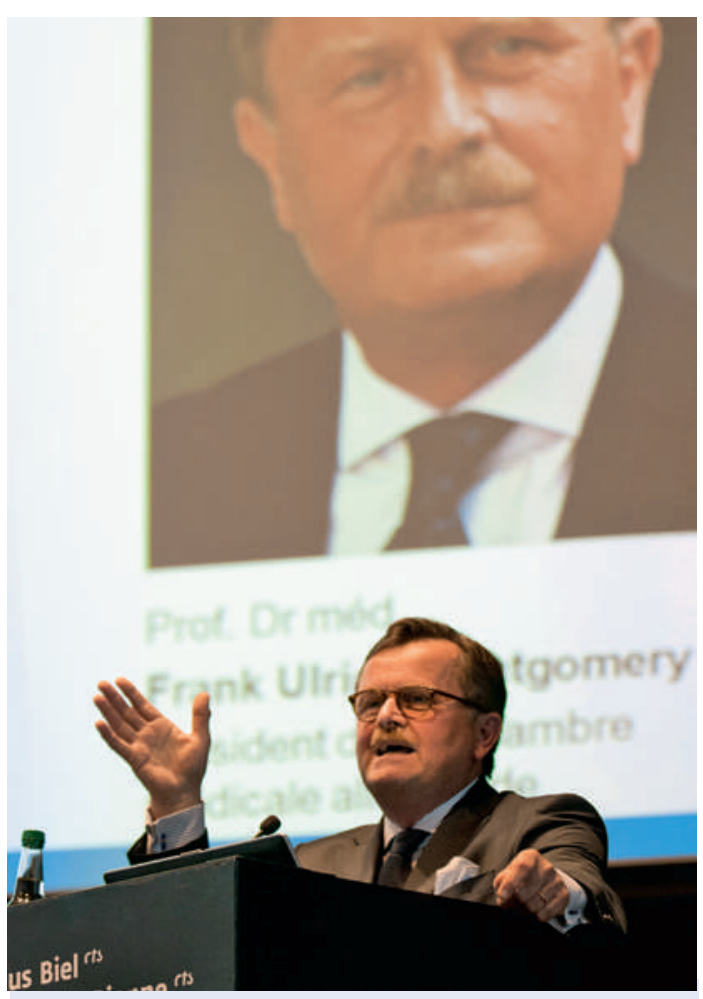

Überraschungsgast an der Ärztekammer war Prof. Dr. Frank Ulrich Montgomery, Präsident der Bundesärztekammer Deutschland.
Überregulierung der Sozialsysteme erstickt Innovation und Fortschritt. Dem deutschen Gesundheitswesen ging es noch nie so gut wie heute, aber es steht vor grossen Herausforderungen. Die Finanzierung der Sozialversicherung, die Bevölkerungsdemographie, die Veränderung der Arztdemographie sowie die neuen von den Ärzten gewünschten Arbeitszeitmodelle bedingen Reformen. Nicht zu verschweigen sind neue Krankheitsbilder wie z.B. Demenz. Dies bedingt, dass heute schon mit dem Aufbau von entsprechenden materiellen und auch personellen Ressourcen begonnen werden muss. Der soziale Konflikt ist programmiert, da die Kinder nicht bereit sein werden, aufgrund dieser Entwicklungen einen höheren Beitragssatz zu bezahlen. Es müssen vernünftige Finanzierungsstrukturen gefunden werden, um der Zukunft gerecht zu werden. Ob die Gesundheitsprämie das richtige Instrument sein soll, wird sich zeigen.

Hinsichtlich der Zukunft zur Finanzierung der gesetzlichen Krankenversicherung hat die Schweiz bereits viel erreicht, und Deutschland liegt diesbezüglich zurück. Deutschland hat mit dem DRG die Zukunft der Krankenhausfinanzierung eingeläutet. Die Fallpauschalen, die die Schweiz mittlerweile auch eingeführt hat, sind noch nicht zu hundert Prozent das richtige System. Deutschland wie auch die Schweiz bewegen sich im gleichen Spannungsfeld: Daseinsversorgung auf der einen Seite, Krankenversorgung auf der anderen Seite. Die politischen und öffentlichen Debatten werden sowohl in Deutschland wie auch in der Schweiz andauern. Zwischen Markt und Ökonomie und den damit verbundenen Zielkonflikten sind alle Beteiligten gefordert, sich Gedanken zu machen und an Lösungen mitzuarbeiten.

Der Präsident dankt Prof. Montgomery bestens für dieses eindrückliche und aufschlussreiche Referat.

\section{Bestätigungswahl Vizepräsident Standeskommission}

Die ÄK hat Dr. med. Urs Strebel in den Gesamterneuerungswahlen 2012 für eine weitere Wahlperiode als Vizepräsident der Standeskommission wiedergewählt. Urs Strebel hat 2013 die statutarisch ordentliche Altersgrenze von 68 Jahren für FMH-Funktionen erreicht. Das Wahlgremium kann gemäss Art. 21 der Statuten die Altersgrenze in Ausnahmefällen hinausschieben.

Antrag ZV: Bestätigung der Funktion von Dr. med. Urs Strebel als Vizepräsident der FMH-Standeskommission bis zur ÄK von Oktober 2014.

Beschluss: Dr. med. Urs Strebel wird mit $110 \mathrm{Ja}$ mit 8 Nein und 6 Enthaltungen als Vizepräsident der FMH-Standeskommission bis zur ÄK Oktober 2014 bestätigt.

Diese Abstimmung ist die erste elektronische Abstimmung der Schweizerischen Ärztekammer. Die elektronischen Resultate werden ebenfalls in die Ab- 
stimmungsliste FMH übertragen, damit eine Sicherheitskopie vorhanden ist.

\section{Bestätigung von gewählten Mitgliedern der DV}

Die ÄK stimmt ebenfalls elektronisch über die Bestätigung der folgenden ordentlichen Delegierten der Delegiertenversammlung ab.

\section{VEDAG}

bisher. Thomas Heuberger, Hilterfingen

neu ab 1.1.2014: Beat Gafner, Niederscherli

VLSS

Bisher: Carlo Moll, Münsterlingen

neu ab 3.10.2013: Karl-Olof Lövblad, Genf

Beschluss: Der Antrag wird mit 111 Ja zu 4 Nein und 9 Enthaltungen gutgeheissen.

\section{Finanzplanung / Finanzcontrolling}

Das Generalsekretariat hat 2011 die Risiken und Schwächen analysiert. Erste Massnahmen wie die Optimierung und Zentralisierung des Budgetprozesses wurden bereits umgesetzt.

Anne-Geneviève Bütikofer/GS präsentiert weitere Instrumente, die seit der letzten ÄK eingeführt wurden. Für die Gewährung einer Finanzhilfe zur Realisierung eines Projekts oder einer Veranstaltung durch die Beauftragung eines Partners oder einer externen Institution muss die FMH zum einen sicherstellen, dass vorher eine finanzielle Analyse stattfindet und zum anderen die anschliessende Kontrolle durchgeführt wird. Das Fehlen eines Rahmens für die finanzielle Unterstützung sowie einer Kontrolle in Bezug auf die
Verwendung der Beiträge und die Verwirklichung des angestrebten Ziels birgt Risiken.

Der ZV hat im Sinne von Art. 50 Abs. 2 Geschäftsordnung folgende Instrumente bereits genehmigt:

6.1 Technische und finanzielle Voranmeldung Hier geht es um die Prüfung der Konformität des Projekts. Sie wird vom Finanzchef bei allen an die FMH gestellten Anträgen auf finanzielle Unterstützung ausgestellt.

\subsection{Bestätigung der Verwendung von Finanz- hilfen (<CHF 10000.-)}

Im Falle einer Finanzhilfe in Höhe von bis zu CHF 10000.- wird eine schriftliche Verpflichtungserklärung des Empfängers verlangt. Der Empfänger ist verpflichtet, die Mittel im Rahmen des angegebenen Gegenstands zu verwenden. Andernfalls behält sich die FMH vor, ihre Finanzhilfe mit unmittelbarer Wirkung zu wiederrufen und keinerlei Zahlung mehr im Falle von gestaffelter Auszahlung zu tätigen oder die Rückerstattung des Gesamt- oder des Teilbetrags zurückzufordern.

\subsection{Leistungsvertrag (>CHF 10000.-)}

Zwischen dem Leistungserbringer und der FMH wird ein Vertrag erstellt. Dieser ist nach Ablauf verlängerbar und ist Gegenstand einer Kontrolle durch den Finanzchef der FMH. Leistungsverträge mit einem Betrag von CHF 100000.- oder mehr für eine einmalige Verpflichtung und von CHF 50000.- oder mehr für eine regelmässig wiederkehrende Verpflichtung sind vorab von der Geschäftsprüfungskommission zu genehmigen.

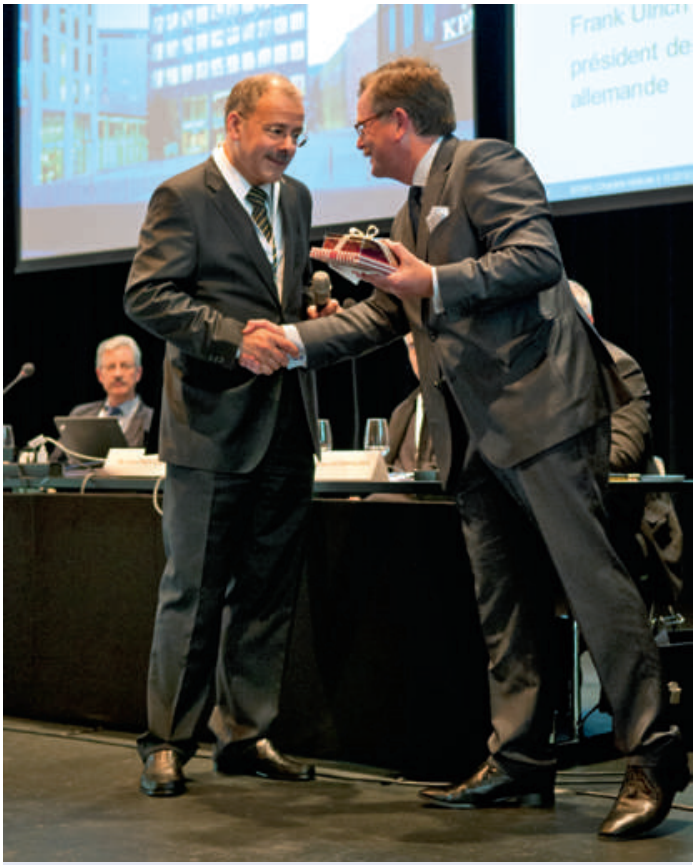

Die berufspolitischen Interessen der Ärzteschaft in der Schweiz und in Deutschland haben zahlreiche Parallelen.

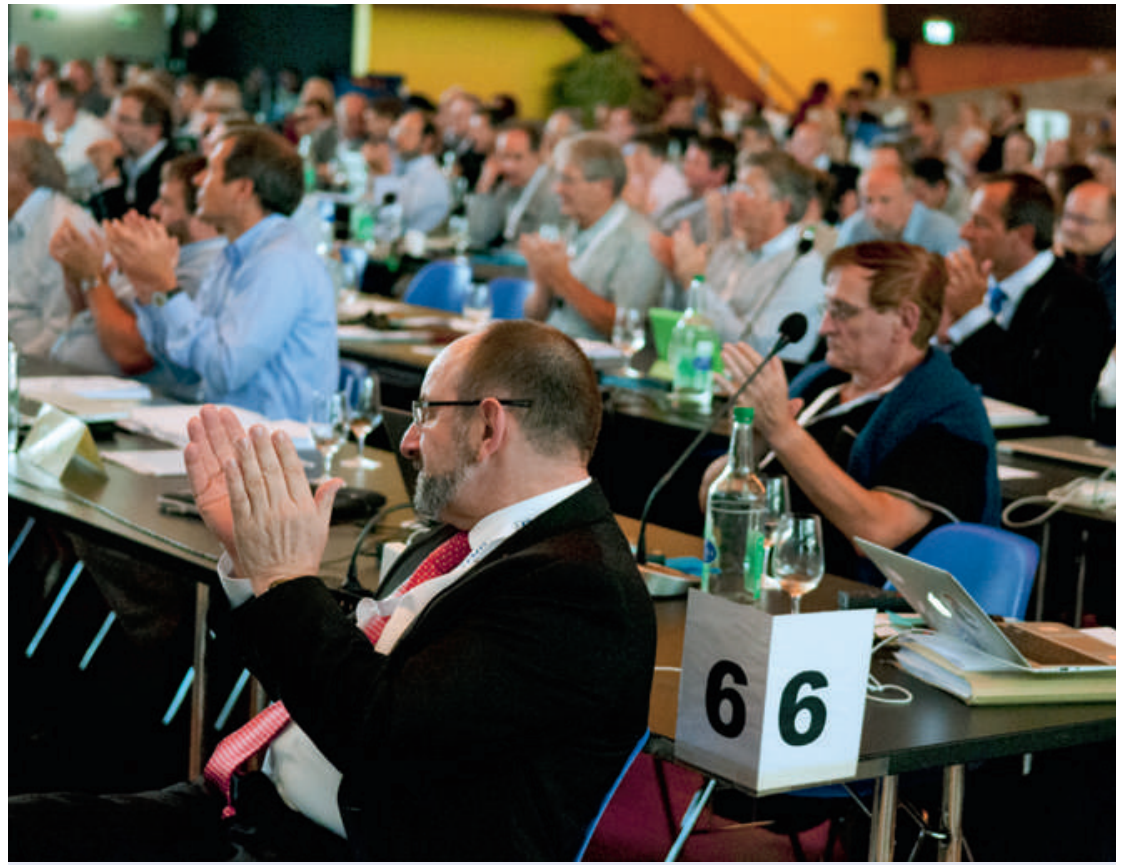

Die Delegierten bedanken sich mit kräftigem Applaus für das spannende und kurzweilige Referat des Überraschungsgastes. 
Weiter informiert die Generalsekretärin über die Einführung eines Projektmanagementtools. Ein erstes Pilotprojekt wurde bereits gestartet.

\section{Budgets 2014 von SIWF, GPK und FMH}

Der Präsident übergibt Christoph Hänggeli/SIWF das Wort. Er nutzt die Gelegenheit, vor der Präsentation des Budgets noch einige Kurzinformationen zu aktuellen Themen zu geben.

- Revision des Medizinalberufegesetzes (MedBG): Der Bundesrat hat viele Anliegen der FMH in die Teilrevision aufgenommen. Ein vollständiges Medizinalberuferegister mit allen Ärztinnen und Ärzten, die den Beruf ausüben, wie es die FMH gefordert hat, wurde jedoch nicht berücksichtigt. Das Bundesamt für Gesundheit stellt sich auf den Standpunkt, dass der Bund hierzu keine Kompetenz habe. Der Bund dürfe nur privatwirtschaftliche Berufsausübungen regeln, nicht aber die Anstellung am öffentlichen Spital. Die FMH hat im Auftrag aller universitären Medizinalberufsverbände bei Prof. Fleiner den Auftrag für ein Gutachten erteilt. In seinem Gutachten kommt er zum Schluss, dass der Bund durchaus die Kompetenz hat, in diesem Bereich legislativ tätig zu werden. Neben einem solchen Register fordert das SIWF weiter, dass die Berufspflichten für alle Ärztinnen und Ärzte gelten, und dass bei der Einführung von neuen eidgenössischen Facharzttiteln die bisher erteilten Titel gleichgestellt sind.

- E-Logbuch:

Das Projekt wurde bereits an der letzten ÄK präsentiert. Das Log-in funktioniert und alle Ärztinnen und Ärzte, ob Mitglieder oder Nichtmitglied, können sich mit Benutzername und Passwort registrieren lassen. Der Zeitfahrplan kann jedoch nicht eingehalten werden. Das Obligatorium für die Einführung wurde auf Mitte 2015 festgesetzt.

- Neue Facharzttitel:

Die zwei neugeschaffenen Facharzttitel Thoraxchirurgie und Gefässchirurgie gab es bereits als Schwerpunkte.

- Projektwettbewerb / Award SIWF:

Die Ausschreibung ist für die Publikation bereit. Für den Wettbewerb werden CHF 100000.- zur Verfügung gestellt. Der Betrag wird für innovative Projekte im Bereich der Weiterbildung ausgeschüttet. Weiter ist auch angedacht, erfolgreiche Weiterbildende mit einem Award auszuzeichnen.

\subsection{Budget 2014 SIWF}

Christoph Hänggeli/SIWF erläutert das Budget 2014. Grundlage für das Budget ist jeweils die Zahl der Titelerteilungen. Gemäss Hochrechnung ist 2014 mit der gleichen Anzahl Titelerteilungen zu rechnen. Das Budget 2014 weist einen Verlust von
CHF 287 000.- auf. Dieser ist auf den Mehraufwand der Projekte, auf interne Verrechnungen sowie Personalkosten zurückzuführen. Auf Ertragsseite ist mit den gleichen Einnahmen zu rechnen, jedoch wird sich die Gebührenreduktion bereits 2014 auf die Ertragsresultate auswirken. Das Defizit wird aus der Auflösung von Rückstellungen getilgt.

Jürg Schlup/FMH dankt für die Ausführungen. Da die Anwesenden keine Fragen haben, wird über den Antrag des SIWF, das Budget 2014 zu genehmigen, abgestimmt.

Beschluss: Das Budget SIWF 2014 wird grossmehrheitlich angenommen.

\subsection{Budget 2014 GPK}

Emanuel Waeber/FMH erläutert das Budget der GPK. Gegenüber dem Budget 2013 gibt es keine speziellen Bemerkungen bzw. Veränderungen.

Daniel Schröpfer/VSAO möchte wissen, weshalb der Mehraufwand von 2012 zu 2014 so gross ist, und ob bereits Reduktionsmassnahmen getroffen wurden.

Adrian Sury/GPK erwidert, dass die Arbeit der GPK schwierig zu budgetieren sei. 2012 war ein Jahr mit einem hohen Mehraufwand, welcher auch auf die personellen Änderungen zurückzuführen ist. Die Übergabe an die neuen Mitglieder hat zu mehr Sitzungen und auch zu Mehrkosten geführt.

Da es keine weiteren Wortmeldungen gibt, wird über das Budget GPK abgestimmt. Der ZV beantragt, das Budget 2014 der GPK zu genehmigen.

Beschluss: Das Budget GPK 2014 wird grossmehrheitlich angenommen.

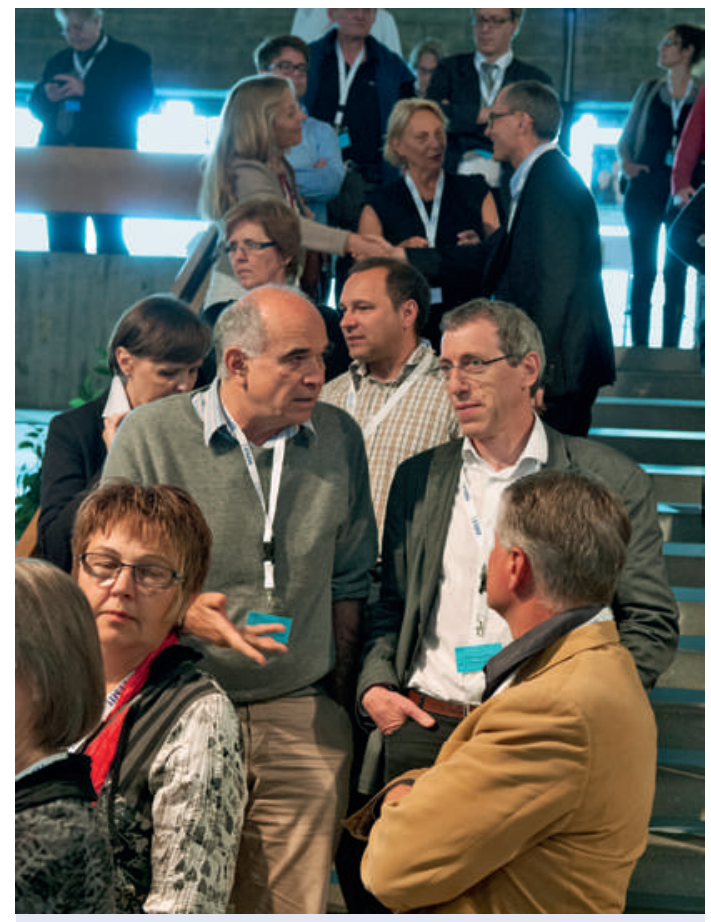

Neben der Plenumsveranstaltung bleibt genügend Raum für den Austausch mit anderen Delegierten. 


\subsection{Budget $2014 \mathrm{FMH}$}

Emanuel Waeber/FMH präsentiert das Budget 2014. Das Verhältnis Ertrag zu den gebundenen Ausgaben lässt 2014 keinen Spielraum zu. Die Reserven werden für die Kernaufgaben der FMH genutzt, nämlich für die Realisierung der Projekte. Das Budget weist einen Verlust von CHF 299000.- aus (ohne SIWF). Die wesentlichen Abweichungen zum Vorjahresbudget sind beim Personalaufwand, und hier insbesondere Löhne Dritter, bei den Projekten sowie bei Lizenzen/ Update/Wartung zu orten. Dank der Auflösung zweckgebundener Rückstellungen kann ein Defizit vermieden werden.

Vincenzo Liguori/TI will wissen, ob bei der FMH ein Personalreglement bestehe, welches die Arbeitszeit, die Über- und Mehrzeit sowie die Ferien regelt. Emanuel Waeber bestätigt ihm, dass diese Reglemente bestehen und jeder Mitarbeitende seine Arbeitszeit elektronisch erfasst.

Adrian Sury/GPK wünscht vor der Abstimmung noch folgenden Hinweis. Gemäss Statuten muss die GPK jeweils zur ÄK einen Bericht abliefern. Dieser beschränkt sich auf die wesentlichen Elemente. Die GPK konnte das Budget 2014 mehrmals mit dem Präsidenten und der Generalsekretärin besprechen. Der hohe Fixkostenanteil, der Anteil der gebundenen Kosten, der ausserordentlich wenig Spielraum für strategische Entwicklung zulässt, bereitet der GPK Sorgen. Um eine Erhöhung der Mitgliederbeiträge zu vermeiden, müssen in nächster Zukunft Lösungen gefunden werden. Der Personalaufwand ist seit der Strukturreformabstimmung in der ÄK 2007 um 30 Prozent gestiegen. Für 2014 sind unter dieser Rubrik wiederum Erhöhungen unvermeidlich. Die beschlossenen Budgetstabilisierungsmassnahmen aus der ÄK 2007 sind teilweise umgesetzt worden. Auch gehen die heute von Anne-Geneviève Bütikofer/GS präsentierten Massnahmen und Instrumente in die von der GPK gewünschte Richtung. Nach Meinung der GPK sollte der ZV sich vorwiegend auf die strategischen Geschäfte konzentrieren und die operativen Aufgaben den Fachpersonen im Generalsekretariat übertragen. Die GPK würde hier schon einen grossen Spareffekt orten. Die GPK hat noch ein anderes strukturelles Problem bei der DV festgestellt. Mit der Einführung von jährlich zwei ÄK stellt sich die GPK die Frage, ob die DV noch jährlich sechsmal tagen muss.

Pierre Vallon/FMPP weist darauf hin, dass sich zurzeit eine Arbeitsgruppe Gedanken über eine Reorganisation der DV macht.

Jürg Schlup/FMH dankt für die Ergänzung. Da es keine weiteren Wortmeldungen gibt, wird über den Antrag des ZV, das Budget 2014 der FMH zu genehmigen, abgestimmt.

Beschluss: Das Budget FMH 2014 wird grossmehrheitlich angenommen.

\subsection{Sonderbeitrag NAKO (NewIndex)}

Ernst Gähler/ZV informiert über den Sonderbeitrag an die Nationale Konsolidierungsstelle NAKO. Die FMH erhebt dafür bei den Mitgliedern der Kategorie 1 und 2 einen zweckgebundenen Sonderbeitrag von CHF 40.-. Die NewIndex-Zahlen werden durch die NAKO für die Tarifverhandlungen von FMH, KKA sowie für die Fachgesellschaften aufbereitet. Die Daten sind einerseits eine wichtige Basis für die Taxpunktwertverhandlungen, anderseits könnte die FMH ohne diese Zahlen nie den Lead bei den Projekten TARVISION und Revision Tarifstruktur übernehmen. Der NAKO-Sonderbeitrag dient vor allem der Basisfinanzierung und der Sicherstellung der Betriebsinfrastruktur und ermöglicht so der Ärzteschaft einen laufenden Zugang zum NAKO-System. Ernst Gähler/ZV bekräftigt nochmals, dass diese Daten für die Tarifrevision entscheidend und unentbehrlich sind und dankt für die Zustimmung.

Da es keine weiteren Wortmeldungen gibt, wird über den Antrag des ZV abgestimmt.

Beschluss: Der Antrag wird grossmehrheitlich angenommen. Die Generalsekretärin wird mit der NewIndex AG einen entsprechenden Leistungsvertrag ausarbeiten.

\subsection{Sonderbeitrag IPI}

François Héritier/SGAM erläutert die Gründe für diesen Antrag sowie die Voraussetzungen für eine elektronische Dokumentation in den Arztpraxen. Noch heute fehlen wesentliche Grundvoraussetzungen, welche zur Folge haben, dass elektronische Daten nicht unter den verschiedenen Softwareprodukten ausgetauscht werden können. Wegen dieser fehlenden Migrierbarkeit sind die einzelnen Ärzte von der einmal gewählten Softwarefirma abhängig. Dies wirkt sich auf die Preise wie auf die Wartungsgebühren aus. Er geht weiter auf die Gründe ein, dem Institut für Praxisinformatik (IPI) das Gesamtmandat für den Bereich der elektronischen Krankengeschichte (eKG) und die konkrete Praxisinformatik zu erteilen. eHealth und die elektronische Krankengeschichte stecken immer noch in den Kinderschuhen. Viele Bedürfnisse der Ärzteschaft bezüglich elektronischer Dokumentation sind noch unerfüllt. Der Bund wie auch eHealthSuisse wollen aber das elektronische Patientendossier möglichst bald einführen. Mit der Übertragung des Gesamtmandats an das IPI würde die Gewähr bestehen, dass die Ärzteschaft die Richtung vorgibt, und die Zusammenarbeit institutionalisiert wird. Die bereits erarbeiteten Grundlagenarbeiten durch die Hausärzte bringen auch für die Spezialisten wie für die Spitäler einen Mehrwert. Er stellt die Vertrauensfrage, ob die Ärzteschaft nun das Institut mit diesem Mandat beauftragen will oder nicht. Die DV wie auch der ZV haben diesem bereits zugestimmt. 
Jürg Schlup/FMH dankt für das klare und dezidierte Votum und erteilt das Wort den Anwesenden.

Adrian Sury/GPK wünscht, den Anwesenden vor der Diskussion noch die Meinung der GPK zu eröffnen. Die GPK hat sich nicht inhaltlich mit der Frage zu beschäftigen, ob das IPI-Projekt nötig und sinnvoll ist, sondern hat zu prüfen, ob das Geschäft statutenkonform abgewickelt wird. Gemäss Art. 32 der Statuten können diverse Organisationen der FMH so auch die SGAM - Antrag auf Traktandierung eines Geschäfts in der ÄK stellen und so theoretisch Präsident, ZV und DV übergehen. Ein solcher Antrag müsste mindestens fünf Wochen vor der ÄK eingereicht werden. Dies ist im vorliegenden Fall nicht geschehen. Die GPK ist hier bereit, auf die Einhaltung der Frist zu verzichten, damit das Geschäft nicht aufs nächste Jahr verschoben wird.

Für die GPK stellt sich jedoch ein anderes Problem. Der Antrag der SGAM ist zweiteilig. Der erste Teil besteht in der Erteilung des Gesamtmandats an das IPI für die eKG. Hier stellt sich die Frage, ob die ÄK gemäss Statuten überhaupt für einen Beschluss zuständig ist. In Art. 30 der Statuten ist keine Zuständigkeit gegeben, gemäss der die ÄK den Abschluss von Verträgen, insbesondere von bilateralen Verträgen mit Leistung und Gegenleistung, zu beschliessen hat. Auch bei der DV gibt es keine entsprechende Kompetenz. Die Generalkompetenz liegt beim ZV, welcher nach Art. 49 Abs. 2 der Statuten auch für dieses Geschäft zuständig ist. Die Finanzierung des Mandats jedoch liegt in der Zuständigkeit der ÄK. Im zugestellten Rundschreiben fehlt jeglicher Hinweis auf den Inhalt des Vertrags zwischen IPI und FMH. Dieses Vorgehen widerspricht den Prinzipien und der Vorgehensweise der FMH.

Damit der Antrag nicht unnötig verzögert wird, schlägt er vor, dass die ÄK heute nur darüber informiert wird. Bevor die Finanzierung nicht langfristig gesichert ist, darf der ZV keinen Vertrag mit dem IPI unterzeichnen. Er erinnert daran, dass in früheren Jahren von einer Anschubfinanzierung der FMH von dreimal CHF 150000.- die Rede war. Heute geht es um einen Betrag von CHF 970000.- für zwei Jahre, ohne die Folgekosten zu kennen. Für die GPK ist dies ein Kerngeschäft der FMH, und somit sollte das Geschäft in einem ZV-Departement beurteilt und behandelt werden.

Aufgrund dieser Voten schlägt Jürg Schlup/Präsident vor, vorerst die Frage der Einreichungsfrist der Antragstellung zu klären. Anschliessend soll über die Erteilung des Gesamtmandats durch die ÄK wie auch über die Genehmigung des Sonderbeitrags entschieden werden.

Bevor die Diskussion eröffnet wird, will der Präsident die Frage der Frist klären und darüber abstimmen.

Beschluss: Die Ärztekammer beschliesst mit $91 \mathrm{Ja}$ gegen 16 Nein auf Eintreten.

Das Geschäft wird somit an der heutigen Ärztekammer behandelt.

Vincenzo Liguori/TI bemängelt, dass bei der Zustellung des Antrags vom 7. September 2013 die französische Übersetzung fehlte. Die Zeit war zu knapp, um innert dieser kurzen Zeit vertieft Stellung zu beziehen. Natürlich schenken alle der Praxisinformatik eine hohe Beachtung, auch wenn die Erfahrungen der vergangenen Jahre zur Vorsicht mahnen. Die eKG ist eigentlich nicht viel mehr als eine ID. Bereits mit der Patientenkarte zeigte sich die Schwierigkeit mit dem Datenaustausch und der fehlenden Komptabilität. Die Vorteile für die Arztpraxen wurden nicht evaluiert, und er beantragt im Namen der Tessiner Gesellschaft, das Geschäft auf die nächste ÄK $\mathrm{zu}$ verschieben, damit in der Zwischenzeit all diese Fragen vertiefter diskutiert und geklärt werden können.

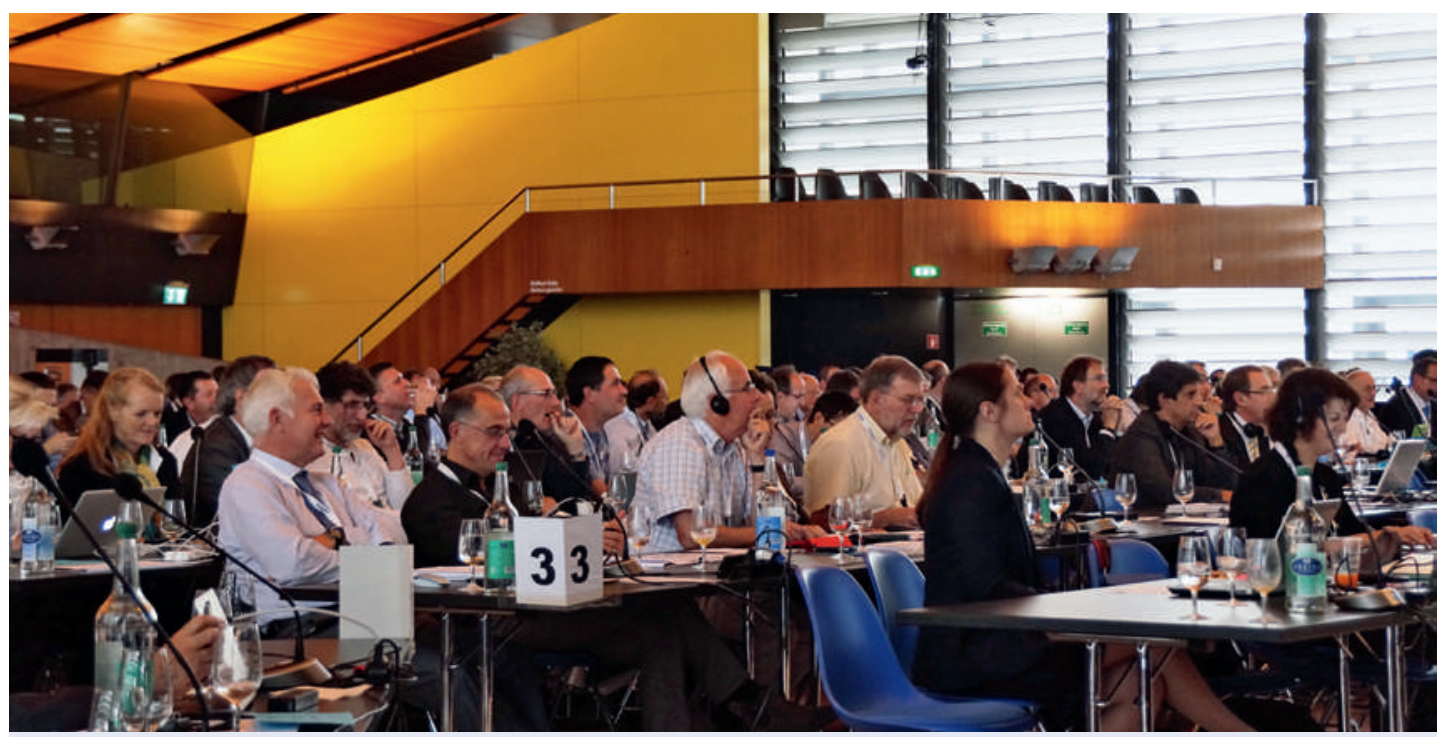

Mit Interesse verfolgen die Anwesenden die Diskussionen der zahlreichen Traktanden. 
Für Jean-Pierre Pavillon/VD sind bis heute Ziele und Aufgaben des IPI unklar. Man kommt nicht um den Eindruck herum, dass hier auch kommerzielle Hintergedanken eine Rolle spielen. Er sieht keine Notwendigkeit, eine solche Institution zu subventionieren. Er stellt den Antrag, dass bei Annahme dieses Geschäftes im Leistungsvertrag explizit festgehalten wird, dass das IPI keine Personalbeziehungen oder irgendwelchen Austausch mit einer kommerziellen Unternehmung pflegen darf.

Pierre-Alain Schneider/GE hält fest, dass die Arbeitsgruppe eHealth sich bereits seit langer Zeit auf politischer Ebene mit diesem Dossier befasst. Die praktische Umsetzung ist jedoch nicht deren Aufgabe. Der nun vorliegende Antrag vom ZV beinhaltet u.a. auch die Delegation von gewissen Mandaten an das IPI. Aber das IPI hat dies abgelehnt. Er findet es inakzeptabel, dass die FMH solche Aktivitäten auslagert. Die Kontrolle über die Aktivitäten ginge damit verloren.

Charles Favrod-Coune/VD kommt nochmals auf das Votum der GPK zurück. Wenn er richtig verstanden hat, will die GPK die Integration des Projekts in ein ZV-Departement. Wenn dem so ist, wünscht er weitere Erklärungen.

Philippe Vuillemin/VD ist aufgrund der 1985 gemachten Erfahrungen und der daraus resultierenden Mehrausgaben der Meinung, dass mit dem IPI die gleiche Erfahrung gemacht werden könnte. Er möchte endlich einmal Resultate sehen und unterstützt die Meinung der GPK, dass heute nur die allgemeine Diskussion geführt wird und der Entscheid auf die nächste ÄK verschoben wird.

Didier Châtelain/GE möchte wissen, ob es bereits Experten gibt, die das IPI leiten werden, und ob es weitere Finanzierungsquellen gibt.

François Héritier/SGAM stellt klar, dass es ein Komitee gibt, welches aus Praxisärzten, einem technischen Direktor sowie einem Vertreter aus der «Konferenz der Kantonalen Ärztegesellschaft (KKA)» besteht. Neue oder weitere Finanzierungsquellen gibt es bis heute nicht.

Jürg Rufener/SGIM appelliert an die Solidarität. Bis heute haben vorwiegend die Hausärzte Schweiz das IPI finanziert und erhebliche Mittel investiert.

Beat Gafner/BE sieht keine andere Möglichkeit, als das IPI mit diesem Gesamtmandat zu beauftragen, und bittet, den Anträgen der SGAM zu folgen.

Für Lars Henrik Frauchiger/VSAO ist das Problem wohl erkannt, jedoch fehlt eine Lösung. Zurzeit wird viel Geld à fonds perdu investiert, ohne einen Gegenwert zu erhalten. Es fehlt ein Businessplan, und die Rollen sind ebenfalls nicht geklärt. Wenn die FMH überhaupt ein solches Institut will, soll es einem ZV-Departement angegliedert werden.

Hervé Spechbach/VSAO befürchtet, dass zu den bereits bestehenden Netzwerken nun ein Institut kreiert wird, was zu Doppelspurigkeiten führen wird.
Franziska Zogg/ZG stellt klar, dass das IPI keine Konkurrenz zu bereits bestehenden Netzwerken sein will. Im Gegenteil, es geht vielmehr darum, heute bereits bestehende kleine Inseln zu verbinden, sei es in der Region mit ein paar angeschlossenen Praxen oder vielleicht auch einer Klinik. Weiter soll die grosse Datenflut in die elektronische Dokumentation integriert werden. Das IPI will diese Schnittstellenarbeiten leisten und die Softwarelieferanten dazu bringen, sich auf eine einzige gemeinsame Übersetzungssprache zu einigen, aber nicht auf ein Programm, das dann alle übernehmen müssen.

Markus Guzek/VSAO will wissen, wofür und für wen das Geld verwendet wird. Da in einem Jahr über die Verlängerung wieder abgestimmt werden soll, sollten die Ziele klar sein, die bis zum nächsten Jahr erfüllt und überprüft werden sollen.

Heinz Bhend/SGAM hat als Delegierter mit dem technischen Leiter IPI diese Fragen vertieft diskutiert. Es geht um die Optimierung der Praxistools. Zurzeit gibt es 65 eHealth-Lösungen für die Praxen, was einen koordinierten Austausch erschwert. Das Ziel von IPI ist und bleibt, hier den Lead zu übernehmen. Wenn diese Aufgabe anderen externen Partnern übertragen wird, kann eine solche Lösung die FMH teuer zu stehen kommen. Für ihn ist die Finanzierung zwingend zu regeln, bevor der ZV beauftragt wird, einen Vertrag auszuarbeiten. Das Geld wird für bereits geleistete Vorarbeiten wie neu auszuhandelnde Verträge mit Softwarefirmen verwendet. Das Argument, dass bis heute noch kein Resultat sichtbar ist, kann er nicht akzeptieren und verweist auf die 2008 entwickelte Roadmap.

Hans Ulrich Iselin/AG macht folgenden Kompromissvorschlag: Der ZV wird ermächtigt, nach Abschluss des Mandatsvertrages einen Beitrag von maximal CHF 30.- einzufordern.

Charles Favrod-Coune/VD will von der GPK die Gründe für einen Transfer des IPI in ein ZV-Departement wissen.

Adrian Sury/GPK vertritt die Meinung, dass Geschäfte mit einer hohen Wichtigkeit für die Ärzteschaft nicht an externe Gesellschaften vergeben werden sollten. Er verweist auf ähnliche Beispiele, die in der Vergangenheit zu Problemen und Verlusten führten. Es gibt eben Kerngeschäfte, die innerhalb der FMH zu bearbeiten sind, damit die Steuerung und Kontrolle auch gewährleistet bleiben.

Peter Wiedersheim/SG ist sich sicher, dass bereits in wenigen Jahren jeder eine eKG besitzen wird. Heute haben bestenfalls 25\% der Praxen die Ausrüstung dazu, die übrigen $75 \%$ tun nun gut daran, endlich JA zu diesem Antrag zu sagen. Bereits seit langem wird über dieses Projekt diskutiert - ohne Erfolg. Dass das IPI damals nicht direkt bei der FMH angesiedelt wurde, ist auf historische Gründe zurückzuführen. Er bittet die Anwesenden, den Antrag zu unterstützen. 
Marc-Henri Gauchat/SMSR erinnert daran, dass e-Health für die Wahl des Topdown-Ansatzes ein Vorwurf gemacht wurde. Beim IPI will man jetzt das Gegenteil, d.h. die Basis soll entscheiden, welche Software sie verwenden will. Das Problem ist, dass es fünf bis sechs Jahre klappt. Danach fehlt eine Garantie, dass die Softwarefirma noch existiert. Und dies wäre der absolute Albtraum. Das e-Dossier betrifft alle Patienten. Wenn man die Industrie nicht zwingt, gewisse Techniken gleich anzuwenden, wird es kaum möglich sein, einfach so die Software zu wechseln. Dies kann zu einem grossen Problem für einen praktizierenden Arzt werden. Im Wallis wurde für das Pilotprojekt INFOMED eine Firma beauftragt, die 50 Kunden hatte. Nach deren Konkurs hatten die Anwender ernsthafte Probleme. Wenn man eine gewisse Sicherheit wünscht, sollte diesem Antrag zugestimmt werden.

Daniel Schröpfer/VSAO weist darauf hin, dass das IPI bereits seit 10 Jahren besteht und bis heute keine konkreten Resultate vorliegen. Der VSAO hat Herrn Zoller im Vorgang zu der ÄK nach den Zielen und dem Businessplan gefragt. Er konnte beide nicht vorweisen. Für ihn ist die Zustimmung zu diesen CHF 30.- indirekt eine Beitragserhöhung, da heute weder ein Nutzen noch ein Resultat sichtbar sind.

Brigitte Muff/ZH würde gerne die Meinung der beiden ZV-Departementsleiter hören.

Gert Printzen/ZV hält fest, dass bei den Diskussionen mit dem IPI und als Resultat der Arbeitsgruppe angeregt wurde, Mandate für einzelne Themen zu bestimmen. Bis heute fehlt jedoch sowohl ein konkretes Konzept als auch ein klares Projekt mit entsprechendem Budget. Eine begründete Stellungnahme des IPI zu diesen Fragen fehlt, was eher darauf hindeutet, dass das IPI einen anderen Weg gehen möchte. Aus diesen Gründen steht er dem Ganzen eher kritisch gegenüber.

Urs Stoffel/ZV stellt bei den Delegierten einen unterschiedlichen Informationsstand fest. Er hat Verständnis, dass die Meinungen zu diesem Projekt gespalten sind. Das eHealth-Departement ist seit langem im Gespräch mit dem IPI. Wenn das Gesamtmandat heute dem IPI übertragen wird, wird das vom ZVDepartement in engster Zusammenarbeit umgesetzt. Das ZV-Departement steht in regelmässigem Kontakt mit dem Koordinationsorgan eHealth des Bundes und der Kantone. Die Diskussionen beziehen sich vorwiegend auf die Schnittstellenprobleme. Ziel wäre eigentlich, eher von Nahtstellen als von Schnittstellen zu sprechen.

Philippe Vuillemin/VD meint, dass nicht die ÄK allein darüber entscheiden kann. Der Beitrag von CHF 30.- ist lächerlich, und dennoch muss den Fachgesellschaften Rechenschaft abgegeben werden, warum man ein Geschäft annimmt, ohne den Nutzen dafür zu kennen. Er ist gegen diese Beitragserhöhung und sieht keine Dringlichkeit, heute schon einen Entscheid zu fällen. Es liegt weder ein Vertrag noch die Finanzierung vor. Da die Hochschule St.Gallen ebenfalls in dieses Projekt involviert ist, möchte er wissen, wie viel diese dafür erhält.

Jürg Rufener/SGIM hält fest, dass sämtliche Beiträge an die Hochschule St. Gallen von Hausärzte Schweiz bezahlt wurden.

Jürg Schlup/Präsident stellt den Ordnungsantrag auf Beendigung der Diskussion. Die bereits gemeldeten Votanten werden noch berücksichtigt.

Beschluss: Dem Ordnungsantrag wird grossmehrheitlich stattgegeben.

Ricardo Torriani/ZH: Die Diskussion ist aus seiner Sicht eher politisch statt sachlich. Seiner Ansicht nach spricht nichts dagegen, das IPI sofort der FMH anzugliedern. Und dies ist ja auch die Forderung der GPK. Die Frage ist nur: Wie ist das möglich, und ist das IPI dazu bereit?

Jürg Schlup/Präsident erinnert daran, dass hier die Anträge der SGAM zu behandeln sind. Weitere Fragen sind heute nicht Gegenstand der gestellten Anträge. Ob das IPI zu einem Transfer bereit ist, müsste geklärt werden. Gemäss GPK ist heute über den Beitrag abzustimmen.

Heinz Bhend/SGAM informiert, dass die Frage der Integration mit dem $\mathrm{ZV}$ verschiedentlich diskutiert wurde. Der momentane Weg ist jedoch eine Kooperation im Hinblick auf die Integration 2016.

Konrad Walter Schiess/SG ist erstaunt, dass der ZV sich in diesem Dossier lediglich auf die Präsentation des IPI abstützt. Die dürftigen Aussagen der ZV-Vertreter sind ungenügend. Wenn das Commitment des ZV heute nicht grösser ist, dann ist er für die Annahme des Antrags, auch im Wissen, dass viele Probleme nicht gelöst sind.

Für Urs Stoffel/ZV wurde das Geschäft an der DV sehr gut vorbereitet und verabschiedet. Eine Arbeitsgruppe hat die verschiedenen Möglichkeiten diskutiert und ist zum Schluss gekommen, über den heute unterbreiteten Antrag abzustimmen. Was die finanziellen Mittel betrifft, kann die FMH das Projekt nicht aus eigener Kraft finanzieren. Es braucht einen Sonderbeitrag. Das IPI hat den Antrag des Sonderbeitrags gestellt; das ganze Prozedere wurde jedoch über die Gremien ZV und DV abgewickelt. Mit dem heute vorgelegten Antrag der SGAM ist auch klar, dass der ZV hinter dem Projekt steht.

Daniel Schröpfer/VSAO hält entgegen, dass die Meinung in der DV uneinheitlich war.

Franziska Zogg/ZG antwortet ihm, dass die Abstimmung in der DV für das Mandat IPI 14:0 war. Es gab wohl Enthaltungen, aber keine Neinstimmen.

Michaël Hagmann/VD verlangt, den Entscheid zu vertagen. Die Komplexität der Informatik- und Softwareprogramme ist derart, dass noch lange Diskussionen geführt werden können. Vermutlich wäre es sinnvoller, eine Übersicht über alle Informatikprojekte zu erstellen. Er stellt den Antrag um Rückweisung an den ZV. Der ZV soll den Vorschlag besser ausarbeiten und der ÄK nochmals unterbreiten. 
Pierre-Alain Schneider/GE unterstützt eine Rückweisung an den ZV.

Monique Lehky Hagen/VS plädiert für die Unterstützung dieses Antrages. Für sie bieten die heute vom Generalsekretariat präsentierten neuen Kontrollinstrumente Gewähr, dass nach Gutheissung dieses Antrages der ZV einen entsprechenden Mandatsvertrag ausarbeiten wird. Und wie bereits erwähnt, wird dieser in einem Jahr an der ÄK wieder überprüft.

Das Abstimmungsprozedere wird festgelegt. Über die Anträge wird wie folgt abgestimmt:

Antrag SGAM: Es wird ein jährlicher Sonderbeitrag IPI von CHF 30.- pro FMH-Mitglied erhoben.

Beschluss: Der Antrag wird mit $53 \mathrm{Ja} \mathrm{zu} 46$ Nein und 6 Enthaltungen angenommen.

Antrag Pavillon: Bei Annahme des Sonderbeitrags muss im Leistungsvertrag die Klausel aufgeführt sein, dass im Falle eines kommerziellen Profits eine vollständige Rückerstattung fällig ist.

Beschluss: Der Antrag wird mit $62 \mathrm{Ja}$ zu 36 Nein und 4 Enthaltungen angenommen.

Antrag Iseli: Der ZV schliesst einen Leistungsvertrag mit dem IPI ab und löst im Anschluss an den Vertragsabschluss den beschlossenen Beitrag von CHF 30.- aus.

Beschluss: Der Antrag wird mit $83 \mathrm{Ja} \mathrm{zu} 12$ Nein und 7 Enthaltungen angenommen.

\section{Hausarztinitiative und Gegenvorschlag}

Die Hausarztinitiative wurde am 27. September 2013 von den Initianten zurückgezogen. Gleichentags hat das Parlament den Gegenvorschlag verabschiedet. Jürg Schlup/Präsident gibt zu bedenken, dass diese Initiative kurz nach ihrer Einreichung von der DV positiv beurteilt wurde. Seither wurden keine Beschlüsse mehr gefasst. Heute geht es nun um die Beurteilung des Gegenvorschlags des Parlaments, um einen neuen Art. 117 a in der Bundesverfassung.
Er übergibt Prof. Peter Tschudi das Wort, welcher die Entwicklung in Bezug auf die Initiative «JA zur Hausarztmedizin» vorstellt. Der Bundesrat hat sich bekanntlich erstmals im Oktober 2010 mit der Initiative auseinandergesetzt und einen direkten Gegenentwurf gegenübergestellt, welcher 2011 vom Bundesrat verabschiedet wurde. Die Initianten haben immer wieder klar festgehalten, welche Bedingungen erfüllt sein müssen, damit sie die Initiative zurückziehen. Prof. Peter Tschudi präsentiert und erläutert den neuen Verfassungsartikel 117a, wie er am 27. September 2013 im Parlament verabschiedet wurde.

Art. 117a in der Bundesverfassung wird wie folgt geändert:

Art. 117a (neu) Medizinische Grundversorgung

1 Bund und Kantone sorgen im Rahmen ihrer Zuständigkeiten für eine ausreichende, allen zugängliche medizinische Grundversorgung von hoher Qualität. Sie anerkennen und fördern die Hausarztmedizin als einen wesentlichen Bestandteil dieser Grundversorgung.

2 Der Bund erlässt Vorschriften über:

a. die Aus- und Weiterbildung für Berufe der medizinischen Grundversorgung und über die Anforderungen zur Ausübung dieser Berufe;

b. die angemessene Abgeltung der Leistungen der Hausarztmedizin.

Ernst Gähler/ZV referiert über die Zusammenhänge der Tarife und der Hausarztinitiative. Mit dem direkten Gegenvorschlag des Parlaments und der bundesrätlichen Zusicherung der finanziellen Besserstellung der Grundversorgung im TARMED sowie der höheren Abgeltung von Laboranalysen sind die zentralen Forderungen des Komitees der Volksinitiative «JA zur Hausarztmedizin» erfüllt. Die FMH begrüsst diese Besserstellung, bedauert aber gleichzeitig, dass mit den gescheiterten TARMED-Verhandlungen der

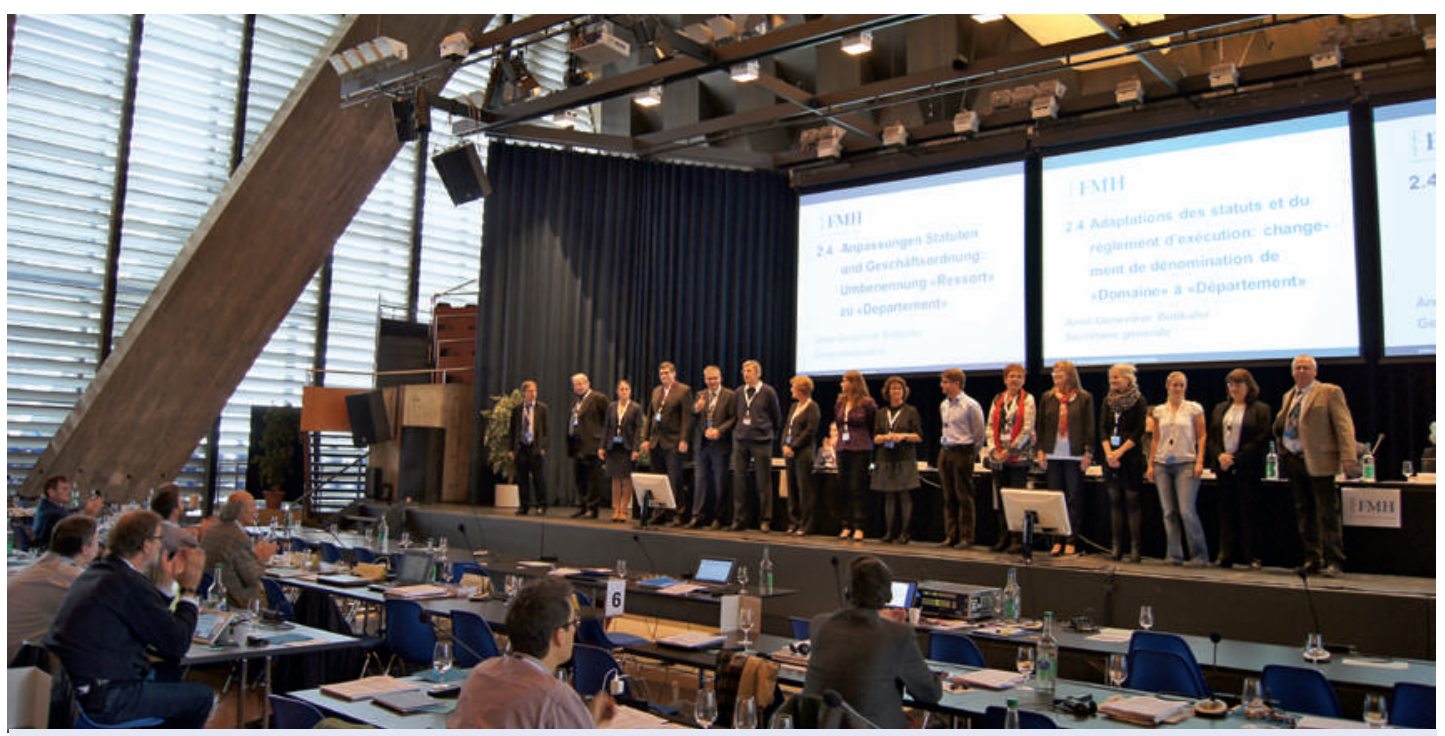

Zahlreiche Helferinnen und Helfer der FMH und des SIWF sorgen für einen reibungslosen Ablauf der Ärztekammer. 


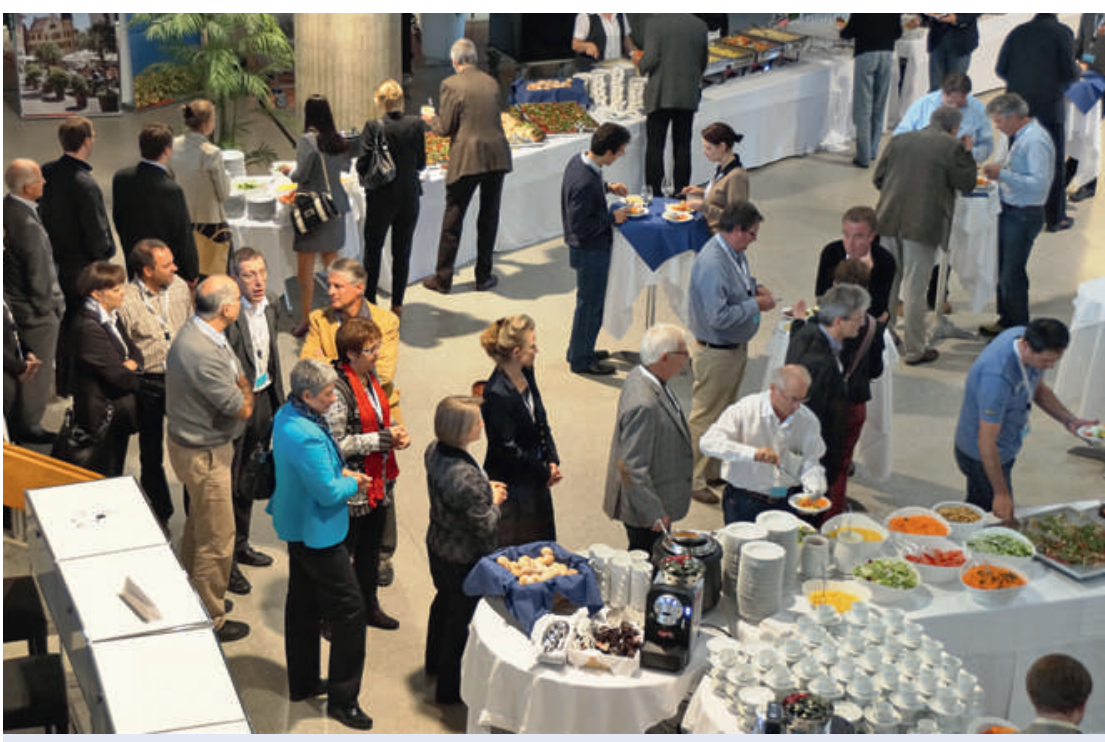

In der Mittagspause stärken sich die Delegierten am Buffet und nutzen die Gelegenheit, sich mit ihren Kollegen auszutauschen. legierten gemäss Art. 34 Abs. 1 der Statuten durchzuführen. Die Delegierten unterstützen diesen Antrag mit 55 Ja zu 9 Nein und 1 Enthaltung. Der ZV wird aufgrund dieses Resultats die weiteren Schritte beraten und beschliessen.

\section{Ergänzung zu Traktandum 8 Hausarztinitiative} und Gegenvorschlag

Im Nachgang zur Ärztekammer wurde eine schriftliche Abstimmung durchgeführt bei den stimmberechtigten Ärztekammer-Delegierten zur Ergänzung der Bundesverfassung mit Art. 117 a (neu) Medizinische Grundversorgung, über welche das Schweizer Stimmvolk voraussichtlich im Mai 2014 entscheidet. Insgesamt waren 200 stimmberechtigte Ärztekammer-Delegierte gebeten, sich zu äussern. Von 150 eingegangenen Stimmzetteln (Stimmbeteiligung von 75\%) waren 149 gültig. Abstimmungsresultat: $110 \mathrm{JA}-$ Stimmen (74\%), 21 NEIN-Stimmen (14\%), 18 Stimmen für Stimmfreigabe (12\%).

Die Ärztekammer spricht sich für eine Ergänzung der Bundesverfassung mit Art. 117 a (neu) Medizinische Grundversorgung aus.

Bundesrat nun die Möglichkeit nutzen kann, Eingriffe in die Tarifstruktur vorzunehmen. Damit ist zum einen die seit drei Jahren laufende TARMEDRevision durch die Tarifpartner FMH, H+ und MTK gefährdet. Es braucht für eine objektive und sachgerechte Bewertung jeder einzelnen Leistung klare Regeln. Die Ärzteschaft muss bei dieser Bewertung der ambulanten medizinischen Leistungen massgeblich mitbestimmen können. Betreffend TARMED hat Bundesrat Berset die Besserstellung der Hausärzte mit 200 Mio. CHF vorgegeben. Die FMH war immer gegen eine lineare Umverteilung von Spezialisten und Grundversorgern, aber für die finanzielle Besserstellung der Haus- und Kinderärzte. Deswegen will die FMH, zusammen mit den Tarifpartnern $\mathrm{H}+$ und der MTK, bis Ende 2015 die laufende Gesamtrevision der TARMED-Tarifstruktur abschliessen.

Zum Zeitpunkt der Abstimmung zu diesem Traktandum sind nur noch 85 Stimmberechtigte anwesend, die ÄK also nicht mehr beschlussfähig. Die Konsultativabstimmung ergibt $61 \mathrm{Ja}$ zu 5 Nein und 19 Enthaltungen.

Hans Ulrich Iselin/AG beantragt dem ZV daraufhin, eine schriftliche Abstimmung unter den ÄK-De-

\section{Aktienkapital-Erhöhung HIN}

Da für eine Abstimmung das Quorum nicht mehr gegeben ist, wird das Geschäft auf die nächste Ärztekammer verschoben.

\section{Bericht aus Zentralvorstand, General- sekretariat und Ressorts}

Aufgrund der fehlenden Zeit wird das Traktandum nicht mehr behandelt.

\section{Bericht des DV-Vorsitzenden}

Aufgrund der fehlenden Zeit wird das Traktandum nicht mehr behandelt.

Jürg Schlup/Präsident beendet die Ärztekammer um 17.00 Uhr und dankt allen Delegierten, den Mitgliedern des ZV, der Generalsekretärin sowie allen Mitarbeitenden des Generalsekretariates für ihre grosse Arbeit zugunsten der Mitglieder. Er appelliert an die ÄK-Delegierten, dass sie als Vertreter unserer Mitglieder auch ihre Verantwortung gegenüber diesen Mitgliedern übernehmen sollen. 


\begin{tabular}{|c|c|c|c|}
\hline $\begin{array}{l}\text { Glossar } \\
\text { ÄK }\end{array}$ & Ärztekammer & ID & $\begin{array}{l}\text { Information und Dokumentation } \\
\text { im Gesundheitswesen }\end{array}$ \\
\hline DRG & Diagnosis Related Groups & IPI & Institut für Praxisinformatik \\
\hline DV & Delegiertenversammlung & MedBG & Medizinalberufegesetz \\
\hline \multirow[t]{2}{*}{ EDK } & \multirow{2}{*}{$\begin{array}{l}\text { Schweizerische Konferenz der } \\
\text { kantonalen Erziehungsdirektoren }\end{array}$} & MTK & Medizinaltarif-Kommission UVG \\
\hline & & NAKO & Nationale Konsolidierungsstelle \\
\hline \multirow[t]{2}{*}{ eHealth } & \multirow{2}{*}{$\begin{array}{l}\text { Zusammenfassung aller elektroni- } \\
\text { schen Gesundheitsdienste }\end{array}$} & SÄZ & Schweizerische Ärztezeitung \\
\hline & & \multirow[t]{2}{*}{ SCG } & \multirow{2}{*}{$\begin{array}{l}\text { Schweizerische Chemische Gesell- } \\
\text { schaft }\end{array}$} \\
\hline eKG & elektronische Krankengeschichte & & \\
\hline \multirow[t]{2}{*}{ e-Logbuch } & \multirow{2}{*}{$\begin{array}{l}\text { elektronisches Logbuch } \\
\text { (Datenbank für Dokumentation } \\
\text { Weiterbildungen) }\end{array}$} & SIWF & $\begin{array}{l}\text { Schweizerisches Institut für } \\
\text { ärztliche Weiter- und Fortbildung }\end{array}$ \\
\hline & & \multirow[t]{2}{*}{ SSO } & \multirow{2}{*}{$\begin{array}{l}\text { Schweizerische Zahnärztegesell- } \\
\text { schaft }\end{array}$} \\
\hline \multirow[t]{2}{*}{ FMH } & \multirow{2}{*}{$\begin{array}{l}\text { Verbindung der Schweizer Ärztin- } \\
\text { nen und Ärzte }\end{array}$} & & \\
\hline & & \multirow[t]{2}{*}{ TARMED } & \multirow{2}{*}{$\begin{array}{l}\text { Tarif Medizin für ambulante } \\
\text { Einzelleistungen }\end{array}$} \\
\hline GPK & Geschäftsprüfungskommission & & \\
\hline GS & Generalsekretärin & TARVISION & Projekt der FMH zur Revision der \\
\hline GST & Gesellschaft Schweizer Tierärztin- & & Tarifstruktur von TARMED \\
\hline & nen und Tierärzte & ZV & Zentralvorstand \\
\hline GUMEK & $\begin{array}{l}\text { Expertenkommission für genetische } \\
\text { Untersuchungen bei Menschen }\end{array}$ & & \\
\hline
\end{tabular}

